\title{
Sopra le estremaloidi relative ad integrali curvilinei dello spazio in forma parametrica.
}

\author{
di Natalia Berruti Onesti (a Pavia).
}

Snnto. - Nella presente Memoria l'Autore si accupa delle estremaloidi relative agli integrali curvilinei dello spazio in forma parametrica

$$
\begin{aligned}
& \mathfrak{g}_{C(2)}^{(2)}=\int_{C(2)} F\left(x(s), y(s), z(s) ; x^{\prime}(s), y^{\prime}(s), z^{\prime}(s) ; u_{2}(s), v_{2}(s), w_{q}(s)\right) d{ }_{s} \\
& \mathfrak{g}_{C(s)}^{(\mathrm{s})}=\int_{C(\mathrm{~s})} F\left(x(s), y(s), z(s) ; x^{\prime}(s), y^{\prime}(s), z^{\prime}(s) ; u_{2}(s), v_{2}(s), w_{2}(s) ; u_{3}(s), v_{3}(s), w_{\mathrm{g}}(s)\right) d s,
\end{aligned}
$$

dimostrando una condizione sufficiente affinchè una curva ordinaria minimante sia un' estrematoide e rilevando alcune proprietà ai esse.

Nel presente lavoro, facendo seguito ad una nostra recente Memoria ( $\left.{ }^{1}\right)$, ci occupiamo delle estremaloidi relative agli integrali curvilinei dello spazio in forma parametrica

$\left(\right.$ a) $\quad \mathfrak{J}_{C^{(2)}}^{(2)}=\int_{C_{(2)}} F^{\prime}\left(x(s), y(s), z(s) ; x^{\prime}(s), y^{\prime}(s), z^{\prime}(s) ; u_{2}(s), v_{2}(s), w_{2}(s)\right) d s$

(b) $\quad \mathfrak{J}_{C^{(8)}}^{(s)}=\int_{C^{(3)}} F\left(x(s), y(s), z(s) ; x^{\prime}(s), y^{\prime}(s), z^{\prime}(s) ; u_{2}(s), v_{2}(s), w_{2}(s) ; u_{3}(s), v_{3}(s), w_{8}(s)\right) d s$,

dove $s$ è la lunghezza dell'arco rettificato, e pertanto $\partial$

$u_{2}(s)=x^{\prime}(s) y^{\prime \prime}(s)-x^{\prime \prime}(s) y^{\prime}(s), \quad v_{2}(s)=y^{\prime}(s) z^{\prime \prime}(s)-y^{\prime \prime}(s) z^{\prime}(s), \quad w_{2}(s)=z^{\prime}(s) x^{\prime \prime}(s)-z^{\prime \prime}(s) x^{\prime}(s)$, $u_{3}(s)=x^{\prime}(s) y^{\prime \prime \prime}(s) \quad x^{\prime \prime \prime}(s) y^{\prime}(s), v_{3}(s)=y^{\prime}(s) z^{\prime \prime \prime}(s)-y^{\prime \prime \prime}(s) z^{\prime}|s|, w_{3}(s)=z^{\prime}(s) x^{\prime \prime \prime}(s)-z^{\prime \prime \prime}(s) x^{\prime}(s) ;$

quindi nella presente ricerca, considerando integrali curvilinei nella forma espressa da $(a)$ e $(b)$, si tiene conto dell' impostazione data da S. CinquinI ( $\left.{ }^{2}\right)$ ai problemi variazionali del secondo e del terzo ordine in forma parametrica affinch ̀̀ il valore dell' integrale sia indipendente dal parametro, e, per sem. plicità, si assume come parametro la lunghezza dell' arco rettificato.

(') N. Berruti Onesti, Sopra le estremali relative ad integrali curvilinei dello spazio in forma parametrica, * Annali di Matematica pura ed applicata", Serie IV, Tomo LII, (1960), pp. $79-106$.

(2) S. CINQuin, Sopra i fondamenti di una classe di problemi variazionali dello spazio, - Rend. del Circolo Matematico di Palermo *, S. II, T. VI (1957), pp. 271-88.

Per il caso in cui il parametro non è la lunghezza dell'arco rettificato, vedi $\$ 2$, n. - $\$ 3$, n. 6 . 
Ricordiamo che recentemente $\left({ }^{3}\right)$ sono state dimostrate alcune condizioni sufficienti affinchè una curva ordinaria minimante l'integrale

$$
\int_{C^{(2)}} F\left(x(s), y(s), x^{\prime}(s), y^{\prime}(s), \vartheta^{\prime}(s)\right) d s
$$

(ove $\vartheta^{\prime}(s)$ rappresenta la curvatura) sia un'estremaloide di ordine 2 , e sono state rilevate alcune proprietà delle estremaloidi relative all'integrale ora scritto.

Nella presente Memoria, alcuni dei risultati conseguiti nelle Note citate in $\left({ }^{3}\right)$ vengono estesi agli integrali $(a)$ e $(b)$; vale a dire, a differenza dai lavori citati in ( ${ }^{(3)}$ nei quali l'Autore, nel caso piano, si è limitato ai problemi variazionali del secondo ordine, noi ci occupiamo dei problemi variazionali del secondo ordine sia di quelli del terzo ordine.

Definite (n. 2) le estremaloidi di ordine 2 e 3 , ha particolare rilievo il teorema, relativo sia al caso del secondo ordine (n. 3) che del terzo ordine (n. 6), in cui viene data una condizione sufficiente affinchè una curva ordi. naria minimante sia un'estremaloide. La dimostrazione, che nel caso del secondo ordine tralasciamo, limitandoci a rilevare qualche caso particolare (n. 4) e ad illustrare il teorema con alcuni esempi (n. 5), è, nel caso del terzo ordine, alquanto laboriosa; nel corso di essa, pur estendendo un proce. dimento che trae origine da ToNELLI $\left({ }^{4}\right)$, si sono dovute superare difficoltà che nascono principalmente dal fatto che, passando dagli integrali curvilinei del piano (c) a quelli dello spazio, le derivate di ordine massimo figurano,

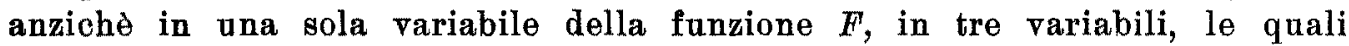
(quando si fa variare singolarmente o l'una o l'altra delle tre funzioni $x(s)$, $\left.y(s), z(s)\left({ }^{4}\right)\right)$ variano tutte e tre.

Tra gli ulteriori risultati contenuti nella presente Memoria, è una condizione sufficiente affinchè un'estremaloide di ordine 2 sia un'estremale (n. $\left.9,2^{\circ}\right)$ ). La dimostrazione di tale teorema ha richiesto nuovi accorgimenti. Infatti, mentre nel caso piano $\left({ }^{5}\right)$ interviene la sola derivata $F_{6^{\prime}}$ e pertanto basta lo stesso ragionamento sviluppato da ToNELLI nel caso del problema del primo

(3) S. Crnquin, Sopra le estremaloidi di una classe di problemi variazionali, "Rend. Accademia Nazionale dei Lincei, S. VIII, vol. XXIII (1957), pp. 116-20. Sopra un teorema relativo alle estremanti di una classe di problemi variazionali, Annali della Scuola Normale Superiore di Pisa ", S. III, vol. XI (1957), pp. 137-47. La seconda di tali Note verrà indicata, nel seguito, brevemente con C. III.

(4) L. Tonelul, Fondamenti di Calcolo delle Variazioni. Due volumi. Vedi vol. II, cap. II, n. 34 a), pp. $98-100$.

(t) Cfr. anche le successive note a piè di pagina $\left({ }^{(2)}\right)$ e (13).

(5) Vedi. S. Cinquint, luogo eit. per primo in $\left({ }^{3}\right)$, n. $1 \mathrm{c}$ ). 
ordine $\left(^{(5}\right)$, nel nostro caso intervengono le tre derivate $F_{w_{2}}, F_{v_{1}}, F_{w_{1}}$, e bisogna ricorrere ad un opportuno artificio.

Al n. 10 infine si estendono brevemente alle estremaloidi di ordine 3 le proprieta rilevate al n. 9 per le estremaloidi di ordine 2.

1. Generalita. - Per tutte le generalità rinviamo ad altri lavori ("), limitandoci a ricordare brevemente alcune definizioni.

a) Sia $F\left(x, y, z ; x^{\prime}, y^{\prime}, z^{\prime} ; u_{2}, v_{2}, w_{2}\right)$ una funzione continua assieme a tutte le proprie derivate parziali del primo ordine in ogni punto $(x, y, z)$ del campo $A$, per ogni terna di numeri reali $x^{\prime}, y^{\prime}, z^{\prime}$ non tutti nulli e per ogni terna di numeri reali $u_{2}, v_{2}, w_{2}$, positivamente omogenea di grado 1 rispetto alle variabili $x^{\prime}, y^{\prime}, z^{\prime}$, e tale che sia $F\left(x, y, z ; 0,0,0 ; u_{2}, v_{2}, w_{2}\right)=0$.

Dicesi curva ordinaria $C^{(2)}$ ogni curva rettificabile

$C^{(2)}: \quad x=x(s), y=y(s), z=z(s), \quad(0 \leq s \leq L)$,

dove $s$ è la lunghezza dell' arco rettificato, per la quale $x(s), y(s), z(s)$ sono funzioni assolutamente continue assieme alle proprie derivate del primo ordine $x^{\prime}(s), y^{\prime}(s), z^{\prime}(s)$, ogni punto $(x(s), y(s), z(s))$, con $0 \leq s \leq L$, appartiene al campo $A$ ed esiste finito l'integrale (di LeBESGOE)

$$
\mathfrak{g}_{C^{(2)}}^{(2)}=\int_{C^{(2)}} F\left(x(s), y(s), z(s) ; x^{\prime}(s), y^{\prime}(s), z^{\prime}(s) ; u_{2}(s), v_{2}(s), w_{2}(s)\right) d s
$$

dove

$$
\begin{gathered}
u_{2}(s)=x^{\prime}(s) y^{\prime \prime}(s)-x^{\prime \prime}(s) y^{\prime}(s), \quad v_{2}(s)=y^{\prime}(s) z^{\prime \prime}(s)-y^{\prime \prime}(s) z^{\prime}(s) \\
w_{2}(s)=z^{\prime}(s) x^{\prime \prime}(s)-z^{\prime \prime}(s) x^{\prime}(s)
\end{gathered}
$$

b) La definizione di curva ordinaria $O^{(3)}$ è analoga a quella di curva ordinaria $C^{(2)}$. Ci limitiamo a rilevare che le funzioni $x(s), y(s), z(s)$, che definiscono una curva ordinaria $C^{(3)}$, sono assolutamente continue assieme alle proprie derivate dei primi due ordini, e tali che esista finito l'integrale (di LEBESGUE)

$$
\mathfrak{J}_{C^{(3)}}^{(s)}=\int_{C^{(3)}} F\left(x(s), y(s), z(s) ; x^{\prime}(s), y^{\prime}(s), z^{\prime}(s) ; u_{2}(s), \quad v_{2}(s), \quad w_{2}(s) ; u_{8}(s), v_{3}(s), w_{3}(s)\right) d s,
$$

$\left({ }^{5}\right)$ Vedi L. TonelLJ, luogo cit. in (4), vol. II, $\mathrm{n}^{\mathrm{i}} 96$ d) e $34 d$ ).

${ }^{(6)}$ Vedi S. Cinquini, Sopra l'esistenza dell'estremo per una classe di integrali curvilinei in forma parametrica, * Annali di Matematica pura ed applicata *, T. XLIX (1960), pp. 25.71. Vedi anche N. Berruti Onest1, luogo eitato in (1), $\$ 1$, n. 1 e $\$ 2$, n. 11. 
dove $F\left(x, y, z ; x^{\prime}, y^{\prime}, z^{\prime} ; u_{2}, v_{2}, w_{2} ; u_{3}, v_{3}, w_{3}\right)$ soddisfa ad ipotesi analoghe a quelle ricordate in a) per la funzione $F\left(x, y, z ; x^{\prime}, y^{\prime}, z^{\prime} ; u_{2}, v_{2}, w_{2}\right)$, le funzioni $u_{2}(s), v_{2}(s), w_{2}(s)$ sono definite dalle (1), ed $\dot{e}$

$$
\begin{gathered}
u_{3}(s)=x^{\prime}(s) y^{\prime \prime \prime}(s)-x^{\prime \prime \prime}(s) y^{\prime}(s), \quad v_{3}(s)=y^{\prime}(s) z^{\prime \prime \prime}(s)-y^{\prime \prime \prime}(s) z^{\prime}(s), \\
w_{3}(s)=z^{\prime}(s) x^{\prime \prime \prime}(s)-z^{\prime \prime \prime}(s) x^{\prime}(s) .
\end{gathered}
$$

2. Definizioni. a) Chiamiamo estremaloide $d i$ ordine 2 relativa alla funzione $F\left(x, y, z ; x^{\prime}, y^{\prime}, z^{\prime} ; u_{2}, v_{2}, w_{2}\right)$ ogni curva ordinaria $C^{(2)}$

$$
x=x(s), y=y(s), z=z(s), \quad(0 \leq s \leq L),
$$

dove $s$ è la lunghezza dell' arco rettificato, tale che le funzioni

$$
\begin{aligned}
& \left(F_{x}\left(x(s) . y(s), z(s) ; x^{\prime}(s), y^{\prime}(s), z^{\prime}(s) ; u_{2}(s), v_{2}(s), w_{2}(s)\right), F_{y}(\ldots), F_{z}(\ldots),\left({ }^{7}\right)\right.
\end{aligned}
$$

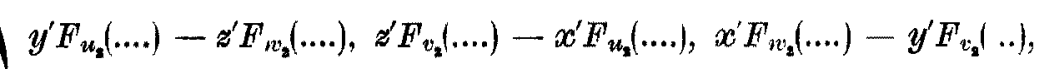

$$
\begin{aligned}
& F_{w^{\prime}}(\ldots)-\left(z^{\prime} v_{2}+2 x^{\prime} u_{2}\right) F_{w_{2}}(\ldots)-3 x^{\prime} v_{2} F_{v_{2}}(\ldots)-\left(y^{\prime} v_{2}+2 x^{\prime} w_{2}\right) F_{w_{3}}(\ldots) \text {, } \\
& F_{y^{\prime}}(\ldots)-\left(z^{\prime} w_{2}+2 y^{\prime} u_{2}\right) F_{u_{2}}(\ldots .)-\left(x^{\prime} w_{2}+2 y^{\prime} v_{2}\right) F_{v_{\mathrm{z}}}(\ldots)-3 y^{\prime} w_{2} F_{w_{2}}(\ldots .), \\
& F_{z^{\prime}}(\ldots)-3 z^{\prime} u_{2} F_{u_{2}}(\ldots)-\left(x^{\prime} u_{2}+2 z^{\prime} v_{2}\right) F_{v_{2}}(\ldots)-\left(y^{\prime} u_{2}+2 z^{\prime} w_{2}\right) F_{w_{3}}(\ldots)
\end{aligned}
$$

risultino integrabili (secondo LEBESGUE) $\left({ }^{\prime}\right)$ sull' intervallo $(0, L)$, e per ogni $s$ di $(0, L)$ siano soddisfatte le equazioni

(3)

$$
\begin{aligned}
\int_{0}^{s} d s \int_{0}^{s} F_{x} d s-\int_{0}^{s}\left[F_{x^{\prime}}-\left(z^{\prime} v_{2}+2 x^{\prime} u_{2}\right) F_{u_{2}}\right. & \left.-3 x^{\prime} v_{2} F_{v_{z}}-\left(y^{\prime} v_{2}+2 x^{\prime} w_{2}\right) F_{v_{z}}\right] d s- \\
& -\frac{d}{d s} \int_{0}^{s}\left(y^{\prime} F_{u_{2}}-z^{\prime} F_{w_{2}}\right) d s=B_{1} s+B_{1}^{\prime}
\end{aligned}
$$

$\int^{s} d s \int_{0}^{s} F_{y} d s-\int_{0}^{s}\left[F_{y^{\prime}}-\left(z^{\prime} w_{2}+2 y^{\prime} u_{2} \mid F_{u_{z}}-\left(x^{\prime} w_{2}+2 y^{\prime} v_{2}\right) F_{v_{z}}-3 y^{\prime} w_{2} F_{w_{2}}\right] d s-\right.$ $\frac{d}{d s} \int_{0}^{s}\left(z^{\prime} F_{v_{2}}-x^{\prime} F_{u_{2}}\right) d \mathrm{~s}=B_{2} s+B_{2}^{\prime}$,

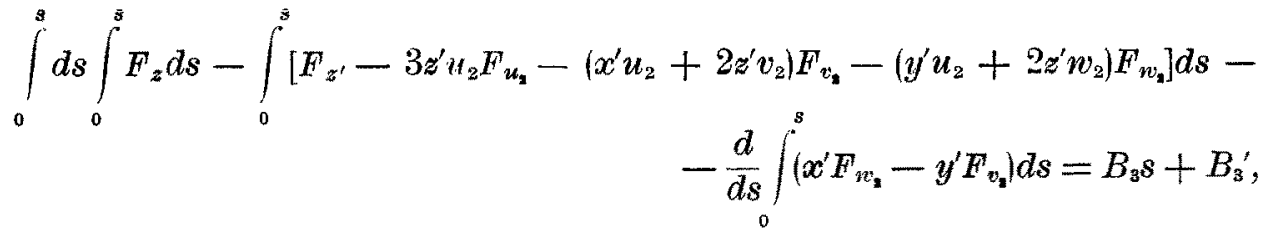

dove $B_{i}, B_{i}^{\prime},(i=1,2,3)$ sono sei costanti.

(7) Indichiamo brevemente, nel segnito del presente capoverso, con $x^{\prime}, y^{\prime}, z^{\prime}, u_{2}, v_{2} . w_{2}$ rispettivamente le funzioni $x^{\prime}(s), y^{\prime}(s), z^{\prime}(s), u_{2}(s), v_{2}(s), w_{2}(s)$.

(") Osserviamo che dallintegrabilitio delle nltime tre delle espressioni ora seritte segue 
b) Posto, per brevità di scrittura,

$$
\left\{\begin{array}{l}
\Phi_{i}=z^{\prime} F_{u_{i}}+x^{\prime} F_{v_{i}}+y^{\prime} F_{w_{i}}, \quad(i=2,3) \\
\Psi_{22}=u_{2} F_{u_{3}}+v_{2} F_{v_{2}}+w_{2} F_{v_{2}} \\
\Psi_{23}=u_{2} F_{u_{3}}+v_{2} F_{v_{3}}+w_{2} F_{w_{3}} \\
\Psi_{33}=u_{3} F_{u_{3}}+v_{3} F_{v_{3}}+w_{3} F_{w_{3}},
\end{array}\right.
$$

chiamiamo estremaloide di ordine 3 relativa alla $\mathrm{funzione}$ $F\left(x, y, z ; x^{\prime}, y^{\prime}, z^{\prime} ; u_{2}, v_{2}, w_{2} ; u_{3}, v_{3}, w_{3}\right)$ ogni curva ordinaria $C^{(3)}$

$$
x=x(s), y=y(s), z=z(s), \quad(0 \leq s \leq L),
$$

dove $s$ è la lunghezza dell'arco rettificato, tale che le funzioni

$\left(4^{\prime}\right)$

$$
\begin{aligned}
& \left.F_{x}(x(s), y(s), z \mid s) ; x^{\prime}(s), y^{\prime}(s), z^{\prime}(s) ; u_{2}(s), v_{2}(s), w_{2}(s) ; u_{3}(s), v_{3}(s), w_{3}(s)\right), F_{y}(\ldots), F_{2}(\ldots)\left({ }^{8}\right), \\
& y^{\prime} F_{w_{\mathrm{a}}}(\ldots .)-z^{\prime} F_{w_{\mathrm{a}}}(\ldots), z^{\prime} F_{v_{\mathrm{g}}}(\ldots)-x^{\prime} F_{u_{\mathrm{a}}}(\ldots), x^{\prime} F_{w_{\mathrm{a}}}(\ldots)-y^{\prime} F_{v_{\mathrm{a}}}(\ldots) \text {, } \\
& y^{\prime} F_{u_{i}}(\ldots)-z^{\prime} F_{w_{3}}(\ldots), z^{\prime} F_{v_{3}}(\ldots)-x^{\prime} F_{w_{3}}(\ldots), x^{\prime} F_{w_{3}}(\ldots)-y^{\prime} F_{v_{3}}(\ldots) \text {, } \\
& F_{x^{\prime}}(\ldots .)-v_{2} \Phi_{2}(\ldots)-v_{3} \Phi_{3}(\ldots)-3 x^{\prime} \Psi_{\mathrm{az}}(\ldots) \text {, } \\
& F_{\nu}(\ldots)-w_{2} \Phi_{2}(\ldots)-w_{3} \Phi_{3}(\ldots)-3 y^{\prime} \Psi_{\text {за }}(\ldots) \text {, } \\
& F_{z^{\prime}}(\ldots)-u_{2} \Phi_{2}(\ldots) \cdots u_{3} \Phi_{3}(\ldots)-3 z^{\prime} \Psi_{\mathrm{s} 3}(\ldots)
\end{aligned}
$$

risultino integrabili (secondo LEBEsGUE) $\left({ }^{\prime \prime}\right)$ sull'intervallo $(0, L)$, e per ogni $s$

l'integrabilità della somma $u_{2} F_{u_{2}}(\ldots)+v_{2} F_{y_{s}}(\ldots)+w_{2} F_{u_{k}}($.$) (e quindi anche quella delle tre$

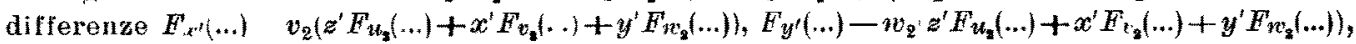
$\left.F_{z^{\prime}}(\ldots)-u_{2}\left(z^{\prime} F_{u_{2}}(.)+.x^{\prime} F_{v_{2}}(\ldots)+y^{\prime} F_{w_{3}}().\right)\right)$; cio si verifica facilmente moltiplicando la prima di tali espressioni per $x^{\prime}$, la seconda per $y^{\prime}$, la terza per $z^{\prime}$ e sommando, tenendo presente l'identità $x^{\prime} v_{2}+y^{\prime} w_{2}+z^{\prime} u_{2}=0$, infine ricordando ehe la funzione $F$ è positivamente omo. genea di grado 1 rispetto a $x^{\prime}, y^{\prime}, z^{\prime}$ e che la (2) è curva ordinaria $C^{(2)}$.

( $\left.{ }^{8}\right)$ Per il seguito del presente capoverso ricordiamo quanto abbiamo detto in (7), $\theta$ facciamo inoltre presente che indichiamo brevemente con $u_{3}, v_{3}, w_{3}$ rispettivamente le funzioni $u_{3}(s), v_{3}(s), w_{3}(s)$.

(8') $L^{\prime}$ integrabilità della funzione $\Psi_{22}(\ldots)$ scende dall'integrabilità delle tre differenze $y^{\prime} F_{w_{2}}(\ldots)-z^{\prime} F_{w_{2}}(\ldots), z^{\prime} F_{v_{2}}(\ldots)-x^{\prime} F_{u_{2}}(\ldots), x^{\prime} F_{w_{2}}(\ldots)-y^{\prime} F_{v_{3}}(\ldots)$, come si verifica facilmente mol. tiplicando la prima di tali differenze per $x^{\prime \prime}$, la seconda per $y^{\prime \prime}$, la terza per $z^{\prime \prime}$ e sommando; in modo analogo si verifica che dall' integrabilità dello tre differenze $y^{\prime} F_{u_{3}}(\ldots)-z^{\prime} F_{n_{3}}(\ldots)$, $z^{\prime} F_{v_{3}}(\ldots)-x^{\prime} F_{w_{3}}(\ldots), x^{\prime} F_{w_{3}}(\ldots)-y^{\prime} F_{v_{3}}(\ldots)$ segue quella della funzione $\Psi_{23}(\ldots)$. Osserviamo inoltre che dallintegrabilita delle ultime tre espressioni delle $\left(4^{\prime}\right)$ scende quella della funzione $\Psi_{33}(\ldots)$ (e quindi anche quella di $E_{w^{\prime}}(\ldots)-v_{2} \Phi_{2}(.)-.v_{3} \Phi_{3}(\ldots), E_{y^{\prime}}(.)-.w_{2} \Phi_{2}(.)-.w_{3} \Phi_{3}(\ldots)$, $\left.F_{z^{\prime}}(\ldots)-u_{2} \Phi_{2}(\ldots)-u_{3}{ }^{\prime \prime}{ }_{3}(\ldots)\right)$; cio si verifica in modo analogo a quello seguito in $\left({ }^{7 \prime}\right)$, tenendc inoltre presente l'identita $x^{\prime} v_{3}+y^{\prime} w_{3}+z^{\prime} u_{3} \equiv 0$. 
di $(0, L)$ siano soddisfatte le equazioni $\left({ }^{9}\right)$

(5)

$$
\begin{aligned}
& \int_{0}^{s} d s \int_{0}^{s} d s \int_{0}^{s} F_{x} d s-\int_{0}^{s} d s \int_{0}^{s}\left[F_{x^{\prime}}-v_{2} \Phi_{2}-2 x^{\prime} \Psi_{22}-v_{3} \Phi_{3}-3 x^{\prime} \Psi_{33}-\right. \\
& \left.-3\left(z^{\prime} w_{2}-y^{\prime} u_{2}\right) \Psi_{23}-\frac{1}{R^{2}}\left(y^{\prime} F_{u_{3}}-z^{\prime} F_{w_{3}}\right)\right] d s- \\
& -\int_{0}^{s}\left(y^{\prime} F_{u_{z}}-z^{\prime} F_{w_{1}}+3 x^{\prime} \Psi_{23}\right) d s+\frac{d}{d s} \int_{0}^{s}\left(y^{\prime} F_{u_{3}}-z^{\prime} F_{w_{3}}\right) d s=C_{1} s^{2}+C_{1}^{\prime} s+C_{1}^{\prime \prime}, \\
& \int_{0}^{s} d s \int_{0}^{s} d s \int_{0}^{s} F_{y} d s-\left.\int_{0}^{s} d s \int_{0}^{s}\right|_{y^{\prime}}-w_{2} \Phi_{2}-2 y^{\prime} \Psi_{22}-w_{3} \Phi_{3}-3 y^{\prime} \Psi_{33}- \\
& \left\{\begin{array}{c}
\left.-3\left(x^{\prime} u_{2}-z^{\prime} v_{2}\right) \Psi_{23}-\frac{1}{R^{2}}\left(z^{\prime} F_{v_{3}}-x^{\prime} F_{u_{3}}\right)\right] d s- \\
-\int_{0}^{s}\left(z^{\prime} F_{v_{2}}-x^{\prime} F_{u_{4}}+3 y^{\prime} \Psi_{23}\right) d s+\frac{d}{d s} \int_{0}^{s}\left(z^{\prime} F_{v_{3}}-x^{\prime} F_{u_{3}}\right) d s=C_{2} s^{2}+C_{2}^{\prime} s+C_{2}^{\prime \prime}
\end{array}\right. \\
& \int_{0}^{s} d s \int_{0}^{s} d s \int_{0}^{s} F_{z} d s-\int_{0}^{s} d s \int_{0}^{s}\left[F_{z^{\prime}}-u_{2} \Phi_{2}-2 z^{\prime} \Psi_{22}-u_{3} \Phi_{3}-3 z^{\prime} \Psi_{38}-\right. \\
& \left.-3\left(y^{\prime} v_{2}-x^{\prime} w_{2}\right) \Psi_{23}-\frac{1}{R^{2}}\left(x^{\prime} F_{w_{3}}-y^{\prime} F_{v^{3}}\right)\right] d s- \\
& -\int_{0}^{s}\left(x^{\prime} F_{z v_{3}}-y^{\prime} F_{v_{3}}+3 z^{\prime} \Psi_{2 s}\right) d s+\frac{d}{d s} \int_{0}^{s}\left(x^{\prime} F_{w_{3}}-y^{\prime} F_{v_{3}}\right) d s=C_{3} s^{2}+C_{3}{ }^{\prime} s+C_{3}^{\prime \prime} \text {, }
\end{aligned}
$$

dove

$$
\frac{1}{R}=\sqrt{u_{2}^{2}+v_{2}^{2}+w_{2}^{2}}
$$

è la flessione, e $C_{i}, C_{i}^{\prime}, C_{i}^{\prime \prime},(i=1,2,3)$ sono nove costanti.

(9) La funzione integranda che figura nel secondo termine del primo membro della prima delle (5) si ottiene da quella che, pure sotto il doppio segno di integrale, figura nella prima delle (22) della Memoria citata in (1) (relativa alle estremali) calcolando, in quest'ultima, le $\frac{d}{d s}\left(z^{\prime} v_{2}+2 x^{\prime} u_{2}\right), \frac{d}{d s}\left(3 x^{\prime} v_{2}\right), \frac{d}{d s}\left(y^{\prime} v_{2}+2 x^{\prime} w_{2}\right)$, tenendo conto delle prime due delle (32) e della prima delle (28) del presente lavoro, e ricordando infine le (4). Analogamente la seconda e terza delle (5) si ottengono dalla seconda e terza delle (22) del lavoro citato in (i), tenendo presenti le (32) e, rispettivamente, la seconda e terza delle (28) della presente Memoria. 
3. Teorema. - Si supponga che, in corrispondenza ad ogni campo limi. tato $A_{\lambda}$ appartenente ad $A$, si possano determinare sei numeri positivi $N_{1}, N_{2}$, $N_{3}, M^{\prime}<1, \eta<1, H \geq 1$, in modo che in tutti i punti $(x, y, z)$ di $A_{\lambda}$, per tutte le terne $x^{\prime}, y^{\prime} z^{\prime}$ normalizzate e per ogni coppia $\varphi_{0}, \varphi_{1}$, con $\varphi_{0}^{2}+\varphi_{1}^{2} \leq M^{\prime 2}$, e per qualunque coppia di terne $\left(u_{2}, v_{2}, w_{2}\right),\left(\tilde{u}_{2}, \tilde{v}_{2}, \tilde{w}_{2}\right)$ per cui sia

$$
\left|u_{2}\right|+\left|v_{2}\right|+\left|w_{2}\right| \geq H
$$

$e$

(6) $\left.(1-\eta):\left|u_{2}\right|+\left|v_{2}\right|+\left|w_{2}\right|\right\} \leq\left|\tilde{u}_{2}\right|+\left|\tilde{v}_{2}\right|+\left|\tilde{w}_{2}\right| \leq(1+\eta)\left\{\left|u_{2}\right|+\left|v_{2}\right|+\left|w_{2}\right|\right\}$

risulti, posto per brevità

$\Xi_{2}=N_{1} \quad F\left(x, y, z ; x^{\prime}, y^{\prime}, z^{\prime} ; u_{2}, v_{2}, w_{2}\right) \mid+N_{2}\left\{\left|u_{2}\right|+\left|v_{2}\right|+\left|w_{2}\right| \mid+N_{s}\right.$,

$$
\left\{\begin{array}{l}
\left|F_{x}\left(x+\varphi_{0}, y, z ; x^{\prime}+\varphi_{1}, y^{\prime}, z^{\prime} ; \tilde{u}_{2}, \tilde{v}_{2}, \tilde{w}_{2}\right)\right| \leq \Xi_{2} \\
\left|F_{x^{\prime}}(\ldots . .)\right| \leq \Xi_{2} \\
|| u_{2}|+| v_{2}|+| w_{2}||\left\{\left|F_{u_{2}}(\ldots .)\right|+\left|F_{v_{2}}(\ldots .)\right|+\left|F_{w_{2}}(\ldots . .)\right|\right\} \leq \Xi_{2},
\end{array}\right.
$$

dove $\left(x+\varphi_{0}, y, z\right)$ appartiene al campo $A$,

(8) $\left\{\begin{array}{l}\left|F_{y}\left(x, y+\varphi_{0}, z ; x^{\prime}, y^{\prime}+\varphi_{1}, z^{\prime} ; \tilde{u}_{2}, \tilde{v}_{2}, \tilde{w}_{2}\right)\right| \leq \Xi_{2} \\ \left|F_{\nu^{\prime}}(\ldots . .)\right| \leq \Xi_{2} \\ \left.|| u_{2}|+| v_{2}|+| w_{2} \mid\right\}\left\{\left|F_{u_{2}}(\ldots)\right|+\left|F_{v_{2}}(\ldots)\right|+\left|F_{w_{2}}(\ldots .)\right|\right\} \leq \Xi_{2},\end{array}\right.$

dove $\left(x, y+\varphi_{0}, z\right)$ appartiene al campo $A, e$

$$
\left\{\begin{array}{l}
\left|F_{z}\left(x, y, z+\varphi_{0} ; x^{\prime} y^{\prime}, z^{\prime}+\varphi_{1} ; \tilde{u}_{2}, \tilde{v}_{2}, \tilde{w}_{2}\right)\right| \leq \Xi_{2} \\
\left|F_{z^{\prime}}(\ldots .)\right| \leq \Xi_{2} \\
\left.|| u_{2}|+| v_{2}|+| w_{2}|||| F_{u_{2}}(\ldots .)|+| F_{v_{2}}(\ldots . .)|+| F_{w_{2}}(\ldots . .) \mid\right\} \leq \Xi_{2},
\end{array}\right.
$$

dove $\left(x, y, z+\varphi_{0}\right)$ appartiene al campo $A$.

Allora, se

$C_{0}^{(2)}: \quad x=x(s), \quad y=y(s), \quad z=z(s), \quad(0 \leq s \leq L)$

(dove s è la lunghezza dell' arco rettificato) è una curva ordinaria $C^{(2)}$ minimante $\mathfrak{J}_{C^{(2)}}^{(2)}$ in una classe $K^{(2)}$ di curve ordinarie $C^{(2)}$, ogni arco

$\bar{C}_{0}^{(2)}: \quad x=x(s), \quad y=y(s), \quad z=z(s), \quad\left(s_{1} \leq s \leq s_{2}\right)$ 
di $C_{0}^{(2)}$, i cui punti, ad eccezione al più di quelli terminali, siano interni al campo $A$ e di indifferenza rispetto ad $A$ e a $K^{(2)} \grave{e}$ un'estremaloide di ordıne 2 relativa alla funzione $F$, cioè per ogni $s$ di $\left(s_{1}, s_{2}\right)$ sono verificate le (3).

Per dimostrare quanto si $\dot{\theta}$ affermato si procede in modo analogo a quello seguito in C. III (n. 2), e, d'altra parie, il teorema ora enunciato si può considerare caso particolare di quello relativo al problema del terzo ordine, che forma oggetto del n. 6. Pertanto tralasciamo la dimostrazione, limitandoci a rilevare che si giunge, -per fissare le idee, alla prima delle (3) considerando la curva

$C_{\gamma}^{(2)}: \quad x=x(s)+\gamma \Omega(s), \quad y=y(s), \quad z=z(s), \quad(0 \leq s \leq L)$,

per la quale sono verificate condizioni analoghe a quelle esposte in C. III (n. 2, p. 141), e dimostrando che esiste finito, per ogni $\gamma$ positivo sufficientemente piccolo, l'integrale

$\mathfrak{g}_{C_{\gamma}^{(2)}}^{(2)}=\int_{0}^{L} F\left(x(s)+\gamma \Omega(s), y(s), z(s) ; x^{\prime}(s)+\gamma \Omega^{\prime}(s), y^{\prime}(s), z^{\prime}(s) ; u_{2 \gamma}(s), v_{2 \gamma}(s), w_{2 \gamma}(s)\right) d s$, dove $\left({ }^{2 \prime}\right)$

$$
\left\{\begin{array}{l}
u_{2 \gamma}(s)=\frac{\left(x^{\prime}(s)+\gamma \Omega^{\prime}(s)\right) y^{\prime \prime}(s)-\left(x^{\prime \prime}(s)+\gamma \Omega^{\prime \prime}(s) \mid y^{\prime}(s)\right.}{\left[\left(x^{\prime}(s)+\gamma \Omega^{\prime}(s)\right)^{2}+y^{\prime 2}(s)+z^{\prime 2}(s)\right]^{\frac{3}{2}}} \\
v_{z \gamma}(s)=\frac{y^{\prime}(s) z^{\prime \prime}(s)-y^{\prime \prime}(s) z^{\prime}(s)}{\left[\left(x^{\prime}(s)+\gamma \Omega^{\prime}(s)\right)^{2}+y^{\prime \prime}(s)+z^{\prime 2}(s)\right]^{\frac{3}{2}}} \\
w_{2 \gamma}(s)=\frac{z^{\prime}(s)\left(x^{\prime \prime}(s)+\gamma \Omega^{\prime \prime}(s)\right)-z^{\prime \prime}(s)\left(x^{\prime}(s)+\gamma{ }^{\prime}(s)\right)}{\left[\left(x^{\prime}(s)+\gamma \Omega^{\prime}(s)\right)^{2}+y^{\prime \prime}(s)+z^{\prime 2}(s)\right]^{3}}
\end{array}\right.
$$

Osservazione I. - È evidente, in virtù della (6), che il teorema del presente $n^{\circ}$ è ancora valido se all' ultima delle (7), (8), (9) si sostituiscono rispettivamente le disuguaglianze

$\left(7^{*}\right)\left\{\left|\tilde{u}_{2}\right|+\left|\tilde{v}_{2}\right|+\left|\tilde{w}_{2}\right|\right\}\left\{\left|\boldsymbol{F}_{u_{2}}\left(x+\varphi_{0}, y, z ; x^{\prime}+\varphi_{1}, y^{\prime}, z^{\prime} ; \tilde{u}_{2}, \tilde{v}_{2}, \tilde{w}_{2}\right)\right|+\left|\boldsymbol{F}_{v_{2}}(\ldots)\right|+\left|\boldsymbol{F}_{w_{2}}(\ldots)\right|\right\} \leq \boldsymbol{\Xi}_{2}$

$\left(8^{*}\right)\left\{\left|\tilde{u}_{2}\right|+\left|\tilde{v}_{2}\right|+\left|\tilde{w}_{2}\right|\right\}\left\{\left|F_{u_{2}}\left(x, y+\varphi_{0}, z ; x^{\prime}, y^{\prime}+\varphi_{1}, z^{\prime} ; \tilde{u}_{2}, \tilde{v}_{2}, \tilde{w}_{2}\right)\right|+\left|F_{v_{2}}(\ldots)\right|+\left|F_{w_{2}}(\ldots)\right| \mid \leq \Xi_{2}\right.$

$\left(9^{*}\right)\left\{\left|\tilde{u}_{2}\right|+\left|\tilde{v}_{2}\right|+\left|\tilde{w}_{2}\right|\right\}\left\{\left|F_{u_{2}}\left(x, y, z+\varphi_{0} ; x^{\prime}, y^{\prime}, z^{\prime}+\varphi_{1} ; \tilde{u}_{2}, \tilde{v}_{2}, \tilde{w}_{2}\right)\right|+\left|F_{v_{2}}(\ldots)\right|+\left|F_{w_{2}}(\ldots)\right|\right\} \leq \mathbf{E}_{2}$.

(9) Per quanto riguarda l'espressione delle funzioni $u_{2}, v_{2}, w_{2}$, quando il parametro da cui dipendono non è la lunghezza dell'arco rettificato, vedi $\mathrm{S}$. Cinquin, luogo cit, in (2), § 2 , n. $\mathbf{3}$. 
Osservazione II. - Si può fare un'osservazione analoga a quella rilevate in C. III (n. 2, Osservazione II).

4. Alcuni casi particolari. - a) Se le funzioni $x(s), y(s), z(s)$ che definiscono la $C_{0}^{(2)}$ hanno la derivata del primo ordine a rapporto incrementale limitato, le disuguaglianze (7), (8) e (9) sono verificate; pertanto si può affermare che ogni arco di curva minimante $\mathscr{J}_{C^{(2)}}^{(2)}$ nel quale le derivate $x^{\prime}(s) y^{\prime}(s)$, $z^{\prime}(s)$ sono a rapporto incrementale limitato è un' estremaloide di ordine 2.

b) Le disuguaglianze (7), (8) e (9) sono pure verificate se, in corrispon. denza ad ogni campo limitato $A_{\lambda}$ appartenente ad $A$, esistono cinque numeri $\alpha>0, M_{1}>0, M_{2}>0, M_{3}>0, m_{0}$, in modo che in tutti $i$ punti (x. $\left.y, z\right) d i A_{\lambda}$, per ogni terna $x^{\prime}, y^{\prime}, z^{\prime}$ normalizzata e per qualunque terna di numeri reali $u_{2}, v_{2}, w_{2}$, sia

$$
\begin{aligned}
& M_{1}\left\{\left|u_{2}\right|+\left|v_{2}\right|+\left|w_{2}\right|\right\}^{\alpha}+m_{0} \leq\left|F\left(x, y, z ; x^{\prime}, y^{\prime}, z^{\prime} ; u_{2}, v_{2}, w_{2}\right)\right| \\
& \qquad \begin{array}{l}
\left|F_{x}\left(x, y, z ; x^{\prime}, y^{\prime}, z^{\prime} ; u_{2}, v_{2}, w_{2}\right)\right| \leq \Theta \\
\left|F_{y}(\ldots)\right| \leq \Theta \\
\left|F_{z}(\ldots)\right| \leq \Theta \\
\left|F_{x^{\prime}}(\ldots)\right| \leq \Theta \\
\left|F_{\nu^{\prime}}(\ldots)\right| \leq \Theta \\
\left|F_{z^{\prime}}(\ldots)\right| \leq \Theta \\
\left.\left.|| u_{2}|+| v_{2}|+| w_{2} \mid\right\}|| F_{u_{z}}(\ldots)|+| F_{v_{2}}(\ldots)|+| F_{w_{2}}(\ldots)\right\} \leq \Theta,
\end{array}
\end{aligned}
$$

dove, per brevità, abbiamo posto

$$
\Theta=M_{2}\left\{\left|u_{2}\right|+\left|v_{2}\right|+\left|w_{2}\right|\right\}^{\alpha}+M_{3}
$$

è ovvio che la (11) è superflua, se $\alpha \leq 1$.

c) Il caso considerato in bl è evidentemente ancora valido, quando siano verificate, in luogo delle (11) e (12), le disuguaglianze che da queste si ottengono sostituendo all' espressione

$$
\left\{\left|u_{2}\right|+\left|v_{2}\right|+\left.\left|w_{2}\right|\right|^{\alpha}\right.
$$

la somma

$$
\left|u_{2}\right|^{\alpha_{1}}+\left|v_{2}\right|^{\alpha_{2}}+\left|w_{2}\right|^{\alpha_{3}}
$$

dove $\alpha_{1}, \alpha_{2}, \alpha_{3}$ sono tre numeri positivi. E anche qui ovvio che la disuguaglianza che si ottiene dalla (11), con la suddetta sostituzione, ̀̀ superflua, quando ognuno dei tre numeri $\alpha_{1}, \alpha_{2}, \alpha_{3}$ e minore od uguale ad 1. 
5. Esempi. - a) La funzione

$$
F=\sqrt{x^{\prime 2}+y^{\prime 2}+z^{\prime 2}}\left(u_{2}^{2}+v_{2}^{2}+w_{2}^{2}\right) e^{\frac{1}{1+u_{2}^{2}+v_{2}^{2}+w_{n}^{2}}}
$$

soddisfa alle condizioni del n. $4, b$ ), per $\alpha=2$.

Ci limitiamo a verificare la (11) e l'ultima delle (12), poiche la verifica delle altre disuguaglianze $\Theta$ immediata oppure più facile a farsi.

Essendo $\sqrt{x^{\prime 2}+y^{\prime 2}+z^{\prime 2}}=1$ e, per ogni terna di numeri reali $u_{2}, v_{2}, w_{2}$,

$$
1<e^{\frac{1}{1+u_{2}^{2}+v_{1}^{2}+w_{v}^{2}}}
$$

e

$$
\left\{\left|u_{2}\right|+\left|v_{2}\right|+\left|w_{2}\right|\right\}^{2} \leq 3\left(u_{2}^{2}+v_{2}^{2}+w_{3}^{2}\right)
$$

si ha evidentemente

$$
\frac{1}{3}\left\{\left|u_{2}\right|+\left|v_{2}\right|+\left|w_{2}\right|\right\}^{2} \leq F
$$

Inoltre, poichè per ogni terna di numeri reali $u_{2}, v_{2}, w_{2}$ è

$$
0<1-\frac{u_{2}^{2}+v_{2}^{2}+w_{2}^{2}}{\left(1+u_{2}^{2}+v_{2}^{2}+w_{2}^{2}\right)^{2}} \leq 1
$$

ed

$$
e^{\frac{1}{1+u_{2}^{2}+v_{2}^{2}+w_{2}^{2}}} \leq e
$$

risulta, a calcoli fatti,

$$
\left.\left\{\left|u_{2}\right|+\left|v_{z}\right|+\left|w_{2}\right|\right\}\left\{\left|F_{u_{2}}\right|+\left|F_{v_{2}}\right|+\left|F_{w_{1}}\right|\right\} \leq 2 e|| u_{2}|+| v_{2}|+| w_{2} \mid\right\}^{2} .
$$

E evidente che sono pare verificate, in questo caso, le disuguaglianze cui si fa cenno nel capoverso $c$ ) dello stesso n. 4, quando sia $\alpha_{1}=\alpha_{2}=\alpha_{3}=2$.

b) Alle ipotesi del n. 3 soddisfano pure le funzioni

$$
\begin{aligned}
& F=\sqrt{x^{\prime 2}+y^{\prime 2}+z^{\prime 2}}\left(u_{2}^{2}+v_{2}^{2}+w_{2}^{2}\right)^{2} \log \left(1+u_{2}^{2}+v_{\mathrm{q}}^{2}+w_{2}^{2}\right) \\
& F=\sqrt{x^{\prime 2}+y^{\prime 2}+z^{\prime 2}} \sqrt[n]{1+\left(u_{z}^{2}+v_{2}^{2}+w_{2}^{2}\right)^{m}}
\end{aligned}
$$

dove $m$ ed $n$ sono interi positivi. 
Per quanto riguarda la prima di tali funzioni, si può verificare, in modo analogo a quello seguito in C. III (n. 3, a)), che sono soddisfatte le (7), (8) e (9) del n. 3.

In merito alla seconda funzione ci limitiamo a rilevare che per $2 m \leq n$ sono verificate le (12) del n. $4, b)$, e per $2 m>n$ sono verificate le (11) e (12) dello stesso capoverso, quando, in entrambi i casi, si assuma $\alpha=\frac{2 m}{n}$. In particolare la (11) e l'ultima delle (12) divengono rispettivamente

$$
\begin{aligned}
& \frac{1}{3^{\frac{m}{n}}}\left\{\left|u_{2}\right|+\left|v_{2}\right|+\left.\left|w_{2}\right|\right|^{\frac{m}{n}}<F\right. \\
& \left.\left\{\left|u_{2}\right|+\left|v_{2}\right|+\left|w_{2}\right|\right\}|| F_{u_{2}}|+| F_{v_{2}}|+| F_{w_{2}} \mid\right\}<\frac{6 m}{n}+\frac{6 m}{n}\left\{\left|u_{2}\right|+\left|v_{2}\right|+\left.\left|w_{2}\right|\right|^{\frac{2 m}{n}}\right.
\end{aligned}
$$

Sono pure verificate le disuguaglianze cui si accenna nel capoverso $c$ ) dello stesso n. 4, quando sia $\alpha_{1}=\alpha_{2}=\alpha_{3}=\frac{2 m}{n}$.

c) La funzione

$$
F=\sqrt{x^{2}+y^{\prime 2}+z^{\prime 2}} \sqrt[n]{1+u_{2}^{2 m}+v_{2}^{2 m}+w_{2}^{2 m}}
$$

dove $m$ ed $n$ sono interi positivi, soddisfa alle condizioni dei capoversi $b$ ) e c) del n. 4.

Ci limitiamo a verificare, tra le disuguaglianze cui si fa cenno nel capoverso c), quelle che si deducono dalla (11) e dall'ultima delle (12), essendo la verifica delle altre disuguaglianze più facile a farsi.

Poichè $\partial$, per ogni terna di numeri reali $u_{2}, v_{2}, w_{2}$ e per qualunque coppia di interi positivi $m$ ed $n,\left({ }^{10}\right)$

$$
\left\{\left|u_{2}\right|^{\frac{2 m}{n}}+\left|v_{2}\right|^{\frac{2 m}{n}}+\left.\left|w_{2}\right|^{\frac{2 m}{n}}\right|^{n} \leq 3^{n-1}\left(u_{2}^{2 m}+v_{2}^{2 m}+w_{z}^{2 m}\right)\right.
$$

si ha

$$
\frac{1}{3^{\frac{n-1}{n}}}\left\{\left|u_{2}\right|^{\frac{2 m}{n}}+\left|v_{2}\right|^{\frac{2 m}{n}}+\left|w_{2}\right|^{\frac{2 m}{n}}\right\}<F
$$

(10) La disuguaglianza che segue si ottiene, come caso particolare, dalla nota disuguaglianza di Jensen. 
Inoltre è

$\left|F_{u_{2}}\right|+\left|F_{v_{2}}\right|+\left|F_{w_{2}}\right|=\frac{2 m}{n}\left(1+u_{2}^{2 m}+v_{2}^{2 m}+w_{2}^{2 m}\right)^{\frac{1}{n}-1}\left\{\left|u_{2}\right|^{2 m-1}+\left|v_{2}\right|^{2 m-1}+\left|w_{2}\right|^{2 m-1}\right\}$

allora, tenendo presente che per qualunque coppia di numeri reali positivi o nulli $A, B$ vale, per ogni coppia di interi positivi $r$ ed $s$, la disuguaglianza

$$
A^{r} B^{s} \leq A^{r+s}+B^{r+s}
$$

si ha

$$
\begin{aligned}
\left\{\left|u_{2}\right|+\left|v_{2}\right|+\left|w_{2}\right|\right\}\left\{\left|F_{u_{3}}\right|+\left|F_{v_{3}}\right|+\left|F_{w_{2}}\right|\right. & \leq \frac{10 m}{n}\left(1+u_{2}^{2 m}+v_{2}^{2 m}+w_{2}^{2 m}\right)^{\frac{1}{n}} \frac{u_{2}^{2 m}+v_{2}^{2 m}+w_{2}^{2 m}}{1+u_{2}^{2 m}+v_{2}^{2 m}+w_{2}^{2 m}}< \\
& <\frac{10 m}{n}+\frac{10 m}{n}\left\{\left|u_{2}\right|^{\frac{2 m}{n}}+\left|v_{2}\right|^{\frac{2 m}{n}}+\left|w_{2}\right|^{\frac{2 m}{n}}\right\} .
\end{aligned}
$$

In modo analogo si possono verificare le (11) e (12) del capoverso b), in cui sia $\alpha=\frac{2 m}{n}$.

6. Tenrema. - Si supponga che, in corrispondenza ad ogni campo limitato $A_{\lambda}$ appartenente ad $A$, e ad ogni numero $M_{0}>0$, si possano determinare sei numeri positivi $N_{1}, N_{2}, N_{3}, M^{\prime}<1, \eta<1, H \geq 1$, in modo che in tutti $i$ punti $(x, y, z)$ di $A_{\lambda}$, per tutte le terne $x^{\prime}, y^{\prime}, z^{\prime}$ normalizzate e per tutte le terne di numeri reali $u_{2}, v_{2}, w_{2}$, con $u_{2}^{2}+v_{2}^{2}+w_{2}^{2} \leq M_{0}^{2}$, per ogni quintupla $\varphi_{0}, \varphi_{1}, \varphi_{2}, \varphi_{3}, \varphi_{4}$, con $\sum_{i=0}^{4} \varphi_{i}{ }^{2} \leq M^{\prime 2}$, e per qualunque coppia di terne $\left(u_{3}, v_{3}, w_{3}\right)$, $\left(\tilde{u}_{3}, \tilde{v}_{3}, \tilde{w}_{3}\right)$ per cui sia

$$
\left|u_{3}\right|+\left|v_{3}\right|+\left|w_{3}\right| \geq H
$$

$\boldsymbol{e}$

(13) $\left.(1-\eta)\left\{\left|u_{3}\right|+\left|v_{3}\right|+\left|w_{3}\right|\right\} \leq\left|\tilde{u}_{3}\right|+\left|\tilde{v}_{3}\right|+\left|\tilde{w}_{3}\right| \leq(1+\eta)|| u_{3}|+| v_{3}|+| w_{3} \mid\right\}$ 
N. Berruti Onesm: Sopra le estremaloidi relative ad integrali, ecc. 231

risulti, posto per brevità

$\Xi_{3}=N_{1}\left|F\left(x, y, z ; x^{\prime}, y^{\prime}, z^{\prime} ; u_{2}, v_{2}, w_{2} ; u_{3}, v_{3}, w_{3}\right)\right|+N_{2}|| u_{3}|+| v_{8}|+| w_{3} \mid+N_{3}$,

$$
\left\{\begin{array}{l}
\left|F_{x}\left(x+\varphi_{0}, y, z ; x^{\prime}+\varphi_{1}, y^{\prime}, z^{\prime} ; u_{2}+\varphi_{2}, v_{2}+\varphi_{3}, w_{2}+\varphi_{4} ; \tilde{u}_{3}, \tilde{v}_{3}, \tilde{w}_{3}\right)\right| \leq \Xi_{3} \\
\left|F_{x^{\prime}}(\ldots . .)\right| \leq \Xi_{3} \\
\left|F_{u_{2}}(\ldots . .)\right| \leq \Xi_{3} \\
\left|F_{v_{2}}(\ldots)\right| \leq \Xi_{3} \\
\left|F_{u_{2}}(\ldots . .)\right| \leq \Xi_{3} \\
\left.\left.|| u_{3}|+| v_{3}|+| w_{3} \mid\right\}|| F_{u_{3}}(\ldots . .)|+| F_{v_{3}}(\ldots .)|+| F_{w_{8}}(\ldots . .) \mid\right\} \leq \Xi_{3},
\end{array}\right.
$$

dove $\left(x+\varphi_{0}, y, z\right)$ appartiene al campo $A$,

$$
\left\{\begin{array}{l}
\left|F_{v}\left(x, y+\varphi_{0}, z ; x^{\prime} y^{\prime}+\varphi_{1}, z^{\prime} ; u_{2}+\varphi_{2}, v_{2}+\varphi_{3}, w_{2}+\varphi_{4} ; \tilde{u}_{3}, \tilde{v}_{3}, \tilde{w}_{3}\right)\right| \leq \Xi_{3} \\
\left|F_{\nu^{\prime}}(\ldots . .)\right| \leq \Xi_{3} \\
\left|F_{u_{3}}(\ldots . .)\right| \leq \Xi_{3} \\
\left|F_{v_{3}}(\ldots . .)\right| \leq \Xi_{3} \\
\left|F_{w_{3}}(\ldots . .)\right| \leq \Xi_{3} \\
\left.\left\{\left|u_{3}\right|+\left|v_{3}\right|+\left|w_{3}\right|\right\}\left|F_{u_{3}}(\ldots . .)\right|+\left|F_{v_{3}}(\ldots . .)\right|+\left|F_{w_{8}}(\ldots . .)\right|\right\} \leq \Xi_{3},
\end{array}\right.
$$

dove $\left(x, y+\varphi_{0}, z\right)$ appartiene al campo $A$

$$
\left\{\begin{array}{l}
\left|F_{z}\left(x, y, z+\varphi_{0} ; x^{\prime}, y^{\prime}, z^{\prime}+\varphi_{2} ; u_{2}+\varphi_{2}, v_{2}+\varphi_{3}, w_{2}+\varphi_{4} ; \tilde{u}_{3}, \tilde{v}_{3}, \tilde{w}_{3}\right)\right| \leq \Xi_{3} \\
\left|F_{z}(\ldots . .)\right| \leq \Xi_{3} \\
\left|F_{u_{3}}(\ldots . .)\right| \leq \Xi_{3} \\
\left|F_{v_{2}}(\ldots .)\right| \leq \Xi_{3} \\
\left|F_{w_{2}}(\ldots . .)\right| \leq \Xi_{3} \\
|| u_{3}|+| v_{3}|+| w_{3}|||| F_{u_{3}}(\ldots .)|+| F_{v_{3}}(\ldots)|+| F_{x v_{3}}(\ldots)|| \leq \Xi_{3},
\end{array}\right.
$$

dove $\left(x, y, z+\varphi_{0}\right)$ appartiene al campo $A$.

Allora, se

$$
C_{0}^{(s)}: \quad x=x(s), \quad y=y(s), \quad z=z(s), \quad(0 \leq s \leq L)
$$

(dove $s$ è la lunghezza dell'arco reltificato) è una curva ordinaria $C^{(3)}$ minimante $\mathfrak{J}_{C^{(3)}}^{(3)}$ in una classe $K^{(3)}$ di curve ordinarie $C^{(3)}$, ogni arco

$$
\bar{C}_{0}^{(a)}: \quad x=x(s), \quad y=y(s), \quad z=z(s), \quad\left(s_{1} \leq s \leq s_{2}\right)
$$


di $C_{0}^{(3)}$, $i$ cui punti, ad eccezione al piì di quelli terminali, siano interni al campo $A$ e di indifferenza rispetto ad $A$ e a $K^{(3)}$, è un'estremaloide di ordine 3 relativa alla funzione $F$, cioè per ogni $s$ di $\left(s_{1}, s_{2}\right)$ sono verificate le equazioni $(5)$.

a) Per dimostrare quanto abbiamo affermato procediamo in modo analogo a quello seguito in C. III (n. 2), limitandoci anche qui, per brevità, a stabilire la prima delle (5).

Si consideri un arco di $C_{0}^{(3)}$

$C^{\prime}$ :

$$
x=x(s), \quad y=y(s), \quad z=z(s), \quad\left(s_{1}{ }^{\prime} \leq s \leq s_{2}{ }^{\prime}\right),
$$

dove $s_{1} \leq s_{1}{ }^{\prime}<s_{2}{ }^{\prime} \leq s_{2}$, tale che ogni suo punto sia interno al campo $A$, e che esista un numero positivo $\rho<1$, con $\rho$ minore della minima distanza dei punti di $C^{\prime}$ dalla frontiera di $A$, in modo che sostituendo a $C^{\prime}$ un qualunque areo di curva ordinaria $C^{(8)}$

$$
x=\bar{x}(\sigma), \quad y=\bar{y}(\sigma), \quad z=\bar{z}(\sigma), \quad\left(\sigma_{1}{ }^{\prime} \leq \sigma \leq \sigma_{2}{ }^{\prime}\right),
$$

dove $\sigma$ è la lunghezza dell'arco rettificato di tale eurva, per il quale sia

$$
\begin{array}{r}
\bar{x}\left(\sigma_{i}^{\prime}\right)=x\left(s_{i}^{\prime}\right), \bar{y}\left(\sigma_{i}^{\prime}\right)=y\left(s_{i}^{\prime}\right), \bar{z}\left(\sigma_{i}^{\prime}\right)=z\left(s_{i}^{\prime}\right), \overline{x^{\prime}}\left(\sigma_{i}^{\prime}\right)=x^{\prime}\left(s_{i}^{\prime}\right), \overline{y^{\prime}}\left(\sigma_{i}{ }^{\prime}\right)=y^{\prime}\left(s_{i}\right), \overline{z^{\prime}}\left(\sigma_{i}^{\prime}\right)=z^{\prime}\left(s_{i}{ }^{\prime}\right) \\
\overline{x^{\prime \prime}}\left(\sigma_{i}^{\prime}\right)=x^{\prime \prime}\left(s_{i}^{\prime}\right), \overline{y^{\prime \prime}}\left(\sigma_{i}^{\prime}\right)=y^{\prime \prime}\left(s_{i}{ }^{\prime}\right), \overline{z^{\prime \prime}}\left(\sigma_{i}^{\prime}\right)=z^{\prime \prime}\left(s_{i}\right), \quad(i=1,2),
\end{array}
$$

ed appartenente all'intorno $(\rho)^{3}$ di $C^{\prime}$, non si esca dalla classe $K^{(3)}$.

Sia $A_{\lambda}$ il campo (limitato) costituito dai punti di $A$ che distano non più di $\rho$ da almeno un punto di $C^{\prime}$ e, considerate le funzioni $u_{2}(s), v_{2}(s), w_{2}(s)$ definite dalle (1) del n. 1 , e relative alla $C_{0}^{(3)}$, si assuma per $M_{0}$ il massimo di $\left|u_{2}(s)\right|+\left|v_{2}(s)\right|+\left|w_{2}(s)\right|$ sulla $C_{0}^{(3)}$.

Indichiamo con $\rho^{\prime}$ il minore dei numeri $\rho, M^{\prime}$ (essendo $M^{\prime}, \eta, H, N_{1}, N_{2}, N_{3}$ i numeri che per ipotesi corrispondono ad $A_{\lambda}$ ed a $M_{0}$ l e teniamo presente che $\dot{\theta} \rho^{\prime}<1$.

Osserviamo che, in virtù della continuità di $F_{x}, F_{x^{\prime}}, F_{u_{z}}, F_{v_{z}}, F_{w_{z}}, F_{u_{3}}$, $F_{v_{3}}, F_{w_{3}}$, esiste un numero $\Delta>0$ tale che in ogni punto $(x, y, z)$ di $A_{\lambda}$, per ogni terna $x^{\prime}, y^{\prime}, z^{\prime}$ normalizzata e per ogni terna di numeri reali $u_{2}, v_{2}, w_{2}$, con $u_{2}^{2}+v_{2}^{2}+w_{2}^{2} \leq M_{0}^{2}$, per $\underset{i=1}{\sum} \varphi_{i}^{2} \leq \rho^{\prime 2}$, e per ogni terna $u_{3}, v_{3}, w_{3}$ per cui ̀̀

$$
\left|u_{3}\right|+\left|v_{3}\right|+\left|w_{3}\right| \leq(1+\eta) H
$$

sono verificate le disuguaglianze

$$
\left\{\begin{array}{l}
\left|F_{x x}\left(x, y, z ; x^{\prime}+\varphi_{1}, y^{\prime}, z^{\prime} ; u_{2}+\varphi_{2}, v_{2}+\varphi_{3}, w_{2}+\varphi_{4} ; u_{3}, v_{3}, w_{3}\right)\right| \leq \Delta,\left|F_{x^{\prime}}(\ldots)\right| \leq \Delta, \\
\left|F_{u_{3}}(\ldots)\right| \leq \Delta,\left|F_{v_{z}}(\ldots)\right| \leq \Delta,\left|F_{w_{2}}(\ldots)\right| \leq \Delta \\
\left|F_{u_{3}}(\ldots)\right| \leq \Delta,\left|F_{v_{3}}(\ldots)\right| \leq \Delta,\left|F_{w_{3}}(\ldots)\right| \leq \Delta
\end{array}\right.
$$


mentre per i valori indicati $x, y, z, x^{\prime}+\varphi_{1}, y^{\prime}, z^{\prime}, u_{2}+\varphi_{2}, v_{2}+\varphi_{3}, w_{2}+\varphi_{1}$ e per ogni terna $u_{3}, v_{3}, w_{3}$ per cui è

$$
\left|u_{3}\right|+\left|v_{3}\right|+\left|w_{3}\right| \geq H \geq 1
$$

dall' ultima delle (14) segue, ricordando l'ultima delle (4),

$$
\left\{\begin{array}{l}
\left|\Psi_{33}\left(x+\varphi_{0}, y, z ; x^{\prime}+\varphi_{1}, y^{\prime}, z^{\prime} ; u_{2}+\varphi_{2}, v_{2}+\varphi_{3}, w_{2}+\varphi_{4} ; \tilde{u}_{3}, \tilde{v}_{3}, \tilde{w}_{3}\right)\right| \leq \Xi_{3} \\
\left.\left.\left|v_{3}\right||| F_{u_{3}}(\ldots)|+| F_{v_{3}} \ldots . . .\right)|+| F_{w_{3}}(\ldots) \mid\right\} \leq \Xi_{3} \\
\left|F_{u_{3}}(\ldots)\right| \leq \Xi_{3} \\
\left|F_{v_{3}}(\ldots)\right| \leq \Xi_{3} \\
\left|F_{w_{3}}(\ldots)\right| \leq \Xi_{3},
\end{array}\right.
$$

dove $\left(x+\varphi_{0}, y, z\right)$ appartiene al campo $A$.

Dunque, in virtù delle (14) e poiche $C_{0}^{(8)}$ è una curva ordinaria $C^{(s)}$, ciascuna delle funzioni $\left({ }^{10 \prime}\right)$

$$
\begin{gathered}
F_{x}, F_{x^{\prime}}-v_{2} \Phi_{2}-2 x^{\prime} \Psi_{22}-v_{\mathrm{z}} \Phi_{3}-3 x^{\prime} \Psi_{33}-3\left(z^{\prime} w_{2}-y^{\prime} u_{2}\right) \Psi_{23}-\frac{1}{R^{2}}\left(y^{\prime} F_{u_{3}}-z^{\prime} F_{w 3}\right) \\
y^{\prime} F_{u_{3}}-z^{\prime} F_{w_{8}}+3 x^{\prime} \Psi_{23}, y^{\prime} F_{u_{3}}-z^{\prime} F_{w_{3}}
\end{gathered}
$$

risulta integrabile sull' intervallo $\left(s_{1}{ }^{\prime}, s_{2}{ }^{\prime}\right)$.

Definiamo ora nell'intervallo $(0, L)$ una funzione $\mathbf{Q}(s)$ in modo analogo a quello seguito in C. III (u. 2, p. 141). Sia $E$ il pseudointervallo, di misura $m(E)=s_{2}{ }^{\prime}-s_{1}{ }^{\prime}$, costituito dai punti $s$ di $\left(s_{1}{ }^{\prime}, s_{2}{ }^{\prime}\right)$ nei quali esistono finite le derivate $x^{\prime \prime \prime}: y^{\prime \prime \prime}, z^{\prime \prime \prime}$ ed inoltre l'integrale

$$
\int_{s_{1^{\prime}}}^{s}\left(y^{\prime} F_{u_{3}}-z^{\prime} F^{\prime} w_{3}\right) d s
$$

ha come derivata la funzione integranda, siano $p_{0}, p_{1}, p_{2}, p_{3}$ quattro valori qualsiasi di $E$, con $p_{0}<p_{1}<p_{2}<p_{3}$, e sia $\delta$ un numero reale positivo tale che $2 \delta<p_{i}-p_{1-1},(i=1,2,3)$.

${ }^{\left(0^{\prime}\right)}$ A partire da questo punto indicheremo brevemente con $F_{x}, F_{x^{\prime}}, F_{u_{z}}, F_{v_{k}}, F_{w_{x}}$, $F_{u_{3}}, F_{v_{3}}, F_{w_{3}}, \Phi_{2}, \Phi_{3}, \Psi_{22}, \Psi_{23}, \Psi_{33}$ rispettivamente le derivate parziali della funzione $F$ rispetto ad $x, x^{\prime}, u_{2}, v_{2}, w_{2}, u_{3}, v_{3}, x_{3}$, e le funzioni definite mediante le (4), calcolate in $\left(x(s), y(s), z(s), x^{\prime}(s), y^{\prime}(s), z^{\prime}(s), u_{2}(s), i_{2}(s), w_{2}(s), u_{3}(s), v_{3}(s), w_{3}(s)\right)$, e, salvo qualche eccezione, con $x, y, z, x^{\prime}, y^{\prime}, z^{\prime}$ le funzioni $x(s), y(s), z(s), x^{\prime}(s), y^{\prime}(s), z^{\prime}(s)$, mentre indicazione analoga useremo per le funzioni $u_{2}(s), v_{2}(s), w_{2}(s), u_{3}(s), v_{3}(s), w_{3}(s)$ e per le derivate del secondo e terzo ordine delle $x(s), y(s), z(s)$. 
Siano $A_{0}, A_{1}, A_{2}, A_{3}$ quattro numeri reali qualsiasi non tutti nulli, tali che sia

$$
\left\{\begin{array}{l}
\left|A_{i}\right| \leq 1,(i=0,1,2,3) \\
\sum_{i=0}^{8} A_{i}=0 \\
\left(2 p_{0}+\delta\right) A_{0}+\left(2 p_{1}-\delta\right) A_{1}+\left(2 p_{2}-\delta\right) A_{2}+\left(2 p_{3}-\delta\right) A_{3}=0 \\
p_{0}\left(p_{0}+\delta\right) A_{0}+p_{1}\left(p_{1}-\delta\right) A_{1}+p_{2}\left(p_{2}-\delta\right) A_{2}+p_{3}\left(p_{3}-\delta\right) A_{3}=0
\end{array}\right.
$$

e definiamo in $(0, L)$ una funzione $\omega(s)$ nel seguente modo

$$
\begin{array}{ll}
\omega(s)=A_{0}, & \left(p_{0} \leq s \leq p_{0}+\delta\right), \\
\omega(s)=A_{1}, & \left(p_{1}-\delta \leq s \leq p_{1}\right), \\
\omega(s)=A_{2}, & \left(p_{2}-\delta \leq s \leq p_{2}\right), \\
\omega(s)=A_{3}, & \left(p_{3}-\delta \leq s \leq p_{3}\right), \\
\omega(s)=0 & \text { per ogni altro } s \text { di }(0, L) .
\end{array}
$$

Sia in $(0, L)$

$$
\Omega(s)=\int_{0}^{s} d u \int_{0}^{u} d v \int_{0}^{v} \omega(t) d t=\int_{0}^{s} d u \int_{0}^{u}(u-t) \omega(t) d t
$$

per la quale, єssendo anche

$$
\int_{0}^{s} d u \int_{0}^{u} d v \int_{0}^{v} \omega(t) d t=\frac{1}{2} \int_{0}^{s}(s-t)^{2} \omega(t) d t
$$

si ha, in virtù della prima delle (19) e per il modo in cui è stata definita $\omega(s)$ in $(0, L)$,

$$
|Q(s)| \leq \frac{\left(p_{3}-p_{0}\right)^{9}}{3 !}, \quad\left|\mathbf{Q}^{\prime}(s)\right| \leq \frac{\left(p_{3}-p_{0}\right)^{2}}{2}, \quad\left|\Omega^{\prime \prime}(s)\right| \leq p_{3}-p_{0}, \quad\left|\Omega^{\prime \prime \prime}(s)\right| \leq 1
$$

e, per le ultime tre delle (19),

$$
\mathbf{Q}(s)=\mathbf{\Omega}^{\prime}(s)=\mathbf{\Omega}^{\prime \prime}(s)=0, \quad \text { per } \quad 0 \leq s \leq p_{0}, \quad \text { e } \quad p_{9} \leq s \leq L,
$$


e consideriamo la curva $C_{\Upsilon}^{(3)}:$

$$
x=x(s)+\gamma \Omega(s), \quad y=y(s), \quad z=z(s),
$$

che definita da tre funzioni assolutamente continue assieme alle loro derivate dei primi due ordini.

Osserviamo che, posto

$$
D=\left[\left(x^{\prime}(s)+\gamma^{S^{\prime}}(s)\right)^{2}+y^{\prime 2}(s)+z^{\prime 2}(s)\right]^{\frac{3}{2}},
$$

esiste un numero positivo $\gamma_{1}$, tale che per ogni $|\gamma|<\gamma_{1}$ è

$$
1-\frac{\eta}{2}<\frac{1}{D^{\frac{4}{3}}}<1+\frac{\eta}{2}
$$

$C_{\curlyvee}^{(3)}$ appartiene all' intorno $\left(\rho^{\prime}\right)^{8}$ di $C^{(3)}$, ed $\otimes\left({ }^{11}\right)$

$$
\gamma^{2}\left[\boldsymbol{Q}^{2}(s)+\boldsymbol{Q}^{\prime 2}(s)+G^{2}(\gamma)\right]<\rho^{\prime 2},
$$

dove, posto

$$
T(\gamma)=2+|\gamma| \frac{\left(p_{3}-p_{0}\right)^{2}}{2}
$$

(e ricordando che $M_{0}$ əे il massimo di $\left|u_{2}\right|+\left|v_{2}\right|+\left|w_{2}\right|$ sulla $C_{0}^{(s)}$ ), \& (24) $G(\gamma)=\left(1+\frac{\eta}{2}\right)^{3}\left(p_{3}-p_{0}\right)\left\{M_{0}\left(p_{3}-p_{0}\right)\left[\frac{\left(1+\frac{\eta}{2}\right)^{\frac{3}{4}}}{1+\left(1+\frac{\left.\eta ! \frac{3}{2}\right)^{4}}{4}\right.}\left(3+3|\gamma| \frac{\left(p_{3}-p_{0}\right)^{2}}{4}\left(1+T^{2}(\gamma)\right)+\right.\right.\right.$

$$
\left.\left.\left.+\gamma^{2} \frac{\left(p_{3}-p_{0}\right)^{4}}{8} T^{3}(\gamma)\right)+\frac{\sqrt{2}}{2}\right]+\sqrt{2}\right\} .
$$

(11) Sarebbe sufficiente, in Inogo della (23), la disuguaglianza

$$
\left.\gamma^{2} Q^{2}(s)+Q^{\prime 2}(s)\right]+\left(\left|u_{2 \gamma}(s)-u_{2}(s)\right|+\left|v_{2 \gamma}(s)-v_{2}(s)\right|+\left|w_{2 \gamma}(s)-w_{2}(s)\right|\right)^{2}<p^{\prime 2} ;
$$

ma, nel modo seguito nel testo, con l'introduzione della $G(\gamma)$, la dimostrazione procede in termini più precisi. 
b) Dimostriamo ora che esiste un namero positivo $\gamma_{2}$, con $\gamma_{2} \leq \gamma_{1}$. tale che per ogni $|\gamma|<\gamma_{2}$ esiste finito l'integrale

$\mathfrak{J}_{C_{\curlyvee}^{(3)}}^{(s)}=\int_{0}^{L} F\left(x(s)+\gamma \underline{Q}(s), y(s), z(s) ; x^{\prime}(s)+\gamma^{\prime}(s), y^{\prime}(s), z^{\prime}(s) ; u_{2 \curlyvee}(s), v_{2 \curlyvee}(s), w_{2 \curlyvee}(s) ; u_{3 \curlyvee}(s), v_{3 \curlyvee}(s), w_{3 \curlyvee}(s)\right) d s$,

dove $\left({ }^{12}\right) u_{2 \curlyvee}, v_{2 \curlyvee}, w_{2 \gamma}$ sono espresse dalle $(10)$ del a. 3 , ed è $\left({ }^{13}\right)$

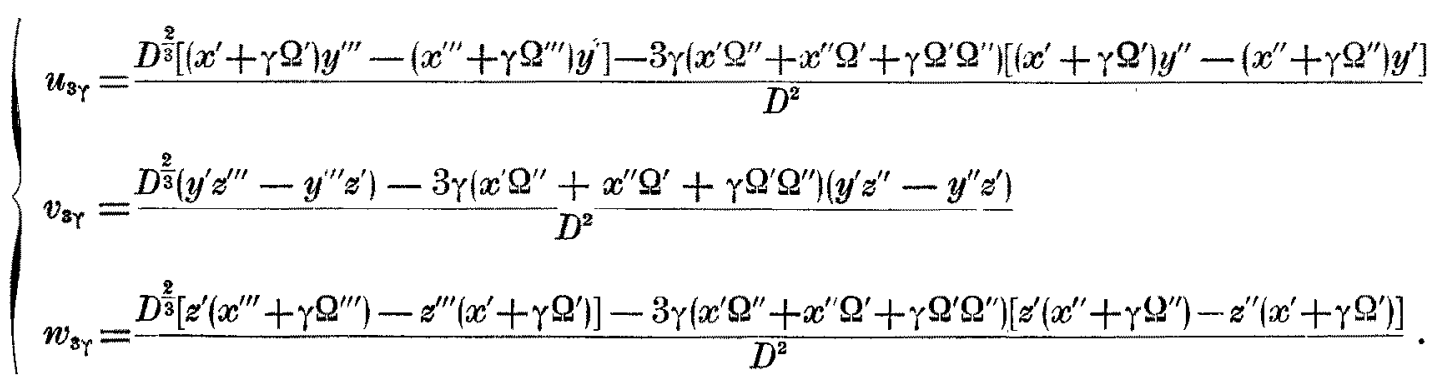

Poichè, in virtù delle (21), per ogni $s$ di $\left(0, p_{0}\right)$ e di $\left(p_{3}, L\right)$ è

$$
u_{2 \curlyvee}=u_{2}, \quad v_{2 \Upsilon}=v_{2}, \quad w_{2 \Upsilon}=w_{2}, \quad u_{3 \Upsilon}=u_{3}, \quad v_{3 \Upsilon}=v_{3}, \quad w_{3 \Upsilon}=w_{3} .
$$

e poichè valgono le (21) possiamo limitarci a considerare l'intervallo $\left(p_{0}, p_{3}\right)$, in cui è, tenuto conto delle ipotesi fatte per la funzione $F$ e le sue derivate parziali del primo ordine,

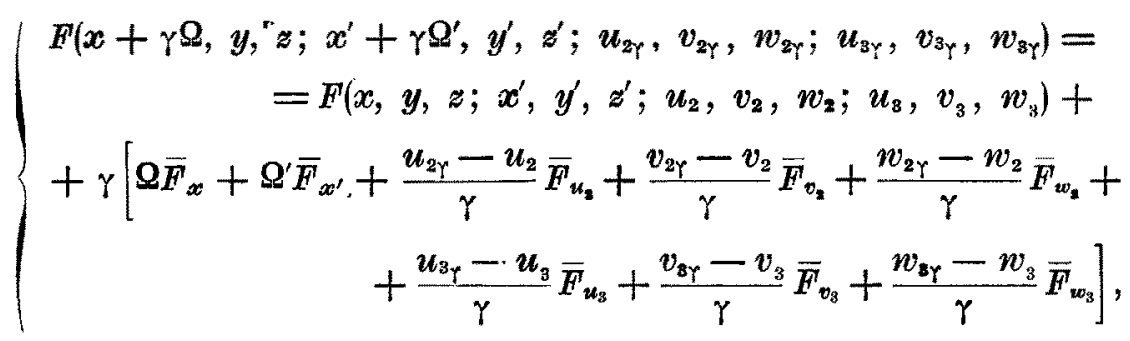

(12) Facoiamo presente che, per brevita, indicheremo nel seguito con $u_{2 \gamma}, v_{2 \gamma}, w_{2 \gamma}$, $u_{8 Y}, v_{3 \gamma}, w_{3 \gamma}, \Omega, Q^{\prime}, Q^{\prime \prime}, \Omega^{\prime \prime \prime}$ rispettivamente le funzioni $u_{2 \gamma}(s), v_{2 \gamma}(s), w_{2 \gamma}(s), u_{8 \Upsilon}(s), v_{3 \gamma}(s), w_{3 \gamma}(s)$, la funzione $Q(s)$ e le sue derivate dei primi tre ordini.

(13) Per quanto riguarda l'espressione delle funzioni $u_{3}, v_{3}, w_{3}$, quando il parametro da oui dipendono non è la lunghezza dell'arco rettificato, vedi $\mathrm{S}$. CINQUINI, Juogo eit. in ( ${ }^{(2)}$, \$ 3 , n. 6 . 
dove il segno posto sopra le derivate parziali della funzione $F$ indica che esse sono calcolate nel punto

$$
\begin{aligned}
& \left(x+\tau_{\gamma} \gamma \mathbf{\Omega}, y, z ; x^{\prime}+\tau_{\gamma} \mathbf{\Omega}^{\prime}, y^{\prime}, z^{\prime} ; u_{2}+\tau_{Y}\left(u_{2 \gamma}-u_{2}\right), v_{2}+\tau_{\gamma}\left(v_{2 \gamma}-v_{2}\right),\right. \\
& \left.w_{2}+\tau_{\Upsilon}\left(w_{2 \gamma}-w_{2}\right) ; u_{3}+\tau_{Y}\left(u_{3 \gamma}-u_{3}\right), v_{3}+\tau_{\Upsilon}\left(v_{3 \gamma}-v_{3}\right), w_{3}+\tau_{\Upsilon}\left(w_{8 \gamma}-w_{3}\right)\right),
\end{aligned}
$$

dove $0<\tau_{Y}<1$.

Rileviamo che dalle (10) del n. 3 segue, ricordando che in tutto $(0, L)$ valgono le identita

$$
\begin{gathered}
\left\{\begin{array}{l}
x^{\prime \prime}=z^{\prime} w_{2}-y^{\prime} u_{2} \\
y^{\prime \prime}=x^{\prime} u_{2}-z^{\prime} v_{2} \\
z^{\prime \prime}=y^{\prime} v_{2}-x^{\prime} w_{2},
\end{array}\right. \\
\left\{\begin{array}{l}
u_{2 \gamma}-u_{2}=u_{2} \frac{1-D^{2}}{D(1+D)}+\frac{\gamma}{D}\left[\left(x^{\prime} u_{2}-z^{\prime} v_{2}\right) \Omega^{\prime}-y^{\prime} \mathbf{Q}^{\prime \prime}\right] \\
v_{2 \gamma}-v_{2}=v_{2} \frac{1-D^{2}}{D(1+D)} \\
w_{2 \gamma}-w_{2}=w_{2} \frac{1-D^{2}}{D(1+D)}+\frac{\gamma}{D}\left[z^{\prime} \mathbf{Q}^{\prime \prime}+\left(x^{\prime} w_{2}-y^{\prime} v_{2}\right) \mathbf{Q}^{\prime}\right]
\end{array}\right.
\end{gathered}
$$

e anche, tenendo presente che dalla (22) segue

$$
\frac{1}{1+D}<\frac{\left(1+\frac{\eta}{2}\right)^{\frac{3}{4}}}{1+\left(1+\frac{\eta}{2}\right)}
$$

e che o

$$
1-D^{2}=-\gamma \Omega^{\prime}\left[6 x^{\prime}+3 \gamma \mathbf{Q}^{\prime}\left(1+\left(2 x^{\prime}+\gamma^{\Omega^{\prime}}\right)^{2}\right)+\gamma^{2} \mathbf{Q}^{\prime 2}\left(2 x^{\prime}+\gamma \mathbf{\Omega}^{\prime}\right)^{8}\right]
$$

e tenuto conto inoltre della seconda e terza delle (20), della (22) e della (24),

$$
\left\{\begin{array}{r}
\left.\left|u_{2 \gamma}-u_{2}\right|+\left|v_{2 \gamma}-v_{2}\right|+\left|w_{2 \curlyvee}-w_{2}\right| \leq \frac{1-D^{2}}{D(1+D)}|| u_{2}|+| v_{2}|+| w_{2} \mid\right\}+ \\
+\frac{|\gamma|}{D} \sqrt{2}\left[\left(\left|u_{2}\right|+\left|v_{2}\right|+\left|w_{2}\right|\right)\left|\Omega^{\prime}\right|+\left|\Omega^{\prime \prime}\right|\right]<|\gamma| G(\gamma) .
\end{array}\right.
$$


Ora osserviamo ohe poichè, tenendo presenti le (28) e la identità $z^{\prime} u_{2}+x^{\prime} v_{2}+y^{\prime} w_{2} \equiv 0$, si ha identicamente in $(0, L)$

$$
\left\{\begin{array}{l}
x^{\prime \prime} u_{2}-z^{\prime \prime} v_{2}=-\frac{1}{R^{2}} y^{\prime} \\
y^{\prime \prime} v_{2}-x^{\prime \prime} w_{2}=-\frac{1}{R^{2}} z^{\prime} \\
z^{\prime \prime} w_{2}-y^{\prime \prime} u_{2}=-\frac{1}{R^{2}} x^{\prime}
\end{array}\right.
$$

dalle (28) si ha, per ogni $s$ di $(0, L)$ in cui esistono finite le derivate $x^{\prime \prime \prime}, y^{\prime \prime \prime}, z^{\prime \prime \prime}$,

$$
\left\{\begin{array}{l}
x^{\prime \prime \prime}=z^{\prime} w_{3}-y^{\prime} u_{3}-\frac{1}{R^{2}} x^{\prime} \\
y^{\prime \prime \prime}=x^{\prime} u_{3}-z^{\prime} v_{3}-\frac{1}{R^{2}} y^{\prime} \\
z^{\prime \prime \prime}=y^{\prime} v_{3}-x^{\prime} w_{3}-\frac{1}{R^{2}} z^{\prime}
\end{array}\right.
$$

Pertanto dalle (25), tenendo presenti le $(28)$ e $\left(32^{\prime}\right)$, e ricordando le $\left(1^{\prime}\right)$ del n. 1 , segue

$$
\begin{aligned}
& u_{3 \varphi}-u_{3}=u_{3}\left(\frac{1}{D^{\frac{4}{3}}}-1\right)+\frac{\gamma}{D^{2}} ! D^{\frac{2}{3}}\left[\left(x^{\prime} u_{3}-z^{\prime} v_{\dot{s}}-\frac{1}{R^{2}} y^{\prime}\right) \mathbf{Q}^{\prime}-y^{\prime} \mathbf{Q}^{\prime \prime \prime}\right]- \\
& \left.-3\left[x^{\prime} \Omega^{\prime \prime}+\left(z^{\prime} w_{2}-y^{\prime} u_{2}\right) \Omega^{\prime}+\gamma \Omega^{\prime} \mathbf{Q}^{\prime \prime}\right]\left[u_{2}+\gamma\left(\left(x^{\prime} u_{2}-z^{\prime} v_{2}\right) \Omega^{\prime}-y^{\prime} \Omega^{\prime \prime}\right)\right]\right\} \\
& v_{3 Y}-v_{3}=v_{3}\left(\frac{1}{D^{\frac{4}{3}}}-1\right)-3 \frac{\gamma}{D^{2}}\left[x^{\prime} \mathbf{Q}^{\prime \prime}+\left(z^{\prime} w_{2}-y^{\prime} u_{2}\right) \mathbf{Q}^{\prime}+\gamma \mathbf{Q}^{\prime} \mathbf{\Omega}^{\prime \prime}\right] v_{2} \\
& w_{3 \curlyvee}-w_{3}=w_{3}\left(\frac{1}{D^{4}}-1\right)+\frac{\gamma}{D^{2}}\left(D^{\frac{2}{3}}\left[z^{\prime} \Omega^{\prime \prime \prime}-\left(y^{\prime} v_{3}-x^{\prime} w_{3}-\frac{1}{R^{2}} z^{\prime}\right) \Omega^{\prime}\right]-\right. \\
& \left.-3\left[x^{\prime} \mathbf{\Omega}^{\prime \prime}+\left(z^{\prime} w_{2}-y^{\prime} u_{2}\right) \mathbf{Q}^{\prime}+\gamma \mathbf{Q}^{\prime} \mathbf{Q}^{\prime \prime}\right]\left[\boldsymbol{w}_{2}+\gamma\left(z^{\prime} \mathbf{Q}^{\prime \prime}-\left(y^{\prime} v_{2}-x^{\prime} w_{2}\right) \Omega^{\prime}\right)\right]\right\}_{1}^{\prime},
\end{aligned}
$$


e anche, indicato, per brevita, rispettivamente con $T_{1}, T_{2}, T_{3}$ il secondo termine del secondo membro della prima, seconda, terza delle (33),

$$
\left\{\begin{array}{l}
u_{3}+\tau_{\gamma}\left(u_{3 Y}-u_{3}\right)=u_{3}\left[1+\tau_{Y}\left(\frac{1}{D^{4}}-1\right)\right]+\tau_{Y} T_{1} \\
v_{3}+\tau_{Y}\left(v_{3 Y}-v_{3}\right)=v_{3}\left[1+\tau_{Y}\left(\frac{1}{D^{4}}-1\right)\right]+\tau_{Y} T_{2} \\
w_{3}+\tau_{Y}\left(w_{3 Y}-w_{3}\right)=w_{3}\left[1+\tau_{Y}\left(\frac{1}{D^{\frac{4}{3}}}-1\right)\right]+\tau_{Y} T_{3} .
\end{array}\right.
$$

Sia ora $\gamma_{2}$ un numero positivo, con $\gamma_{2} \leq \gamma_{1}$, tale che

(35)

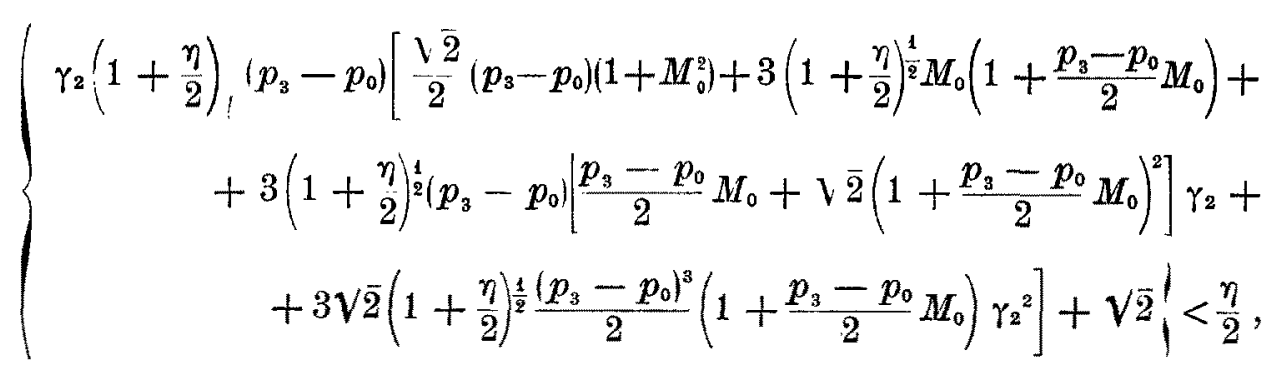

ed osserviamo che per $|\gamma|<\gamma_{2}$, tenute presenti le ultime tre delle (20), la (22), e le (33), si ha

$$
\left\{\begin{array}{r}
\left|T_{1}\right|+T_{2}|+| T_{3} \mid<\gamma_{2}\left(1+\frac{\eta}{2}\right)\left[\frac{\left(p_{3}-p_{0}\right)^{2}}{2} \sqrt{2}\left(\left|u_{3}\right|+v_{3}|+| w_{3} \mid+M_{0}^{2}\right)+\gamma \overline{2}\right]+ \\
+3 \gamma_{2}\left(1+\frac{\eta}{2}\right)^{\frac{3}{2}}\left(p_{3}-p_{0} \mid\left[\frac{p_{3}-p_{0}}{2} M_{0}+\gamma_{2} \frac{\left(p_{3}-p_{0}\right)^{2}}{2}+1\right]\right. \\
\cdot\left[M_{0}+\gamma_{2} \sqrt{2}\left(p_{3}-p_{0}\right)\left(1+\frac{p_{3}-p_{0}}{2} M_{0}\right)\right]
\end{array}\right.
$$

Allora se

$$
\left|u_{3}\right|+\left|v_{3}\right|+\left|w_{3}\right| \leq H
$$

essendo $H \geq 1 \mathrm{e}$, in virtù della (22),

$$
1+\tau_{\Upsilon}\left(\frac{1}{D^{\frac{4}{3}}}-1\right)<1+\frac{\eta}{2},
$$


dalle (34), tenendo presenti la (36) e (35), segue

$$
\left|u_{3}+\tau_{Y}\left(u_{3_{\gamma}}-u_{3}\right)\right|+\mid v_{3}+\tau_{Y}\left(v_{3 \gamma}-v_{3}||+\left|w_{3}+\tau_{\Upsilon}\left(w_{3}-w_{3}\right)\right|<(1+\eta) H ;\right.
$$

pertanto, tenuto conto della (23) e della (31), in virtù delle (17) si ha per ogni $s$ di $\left(p_{0}, p_{3}\right)$

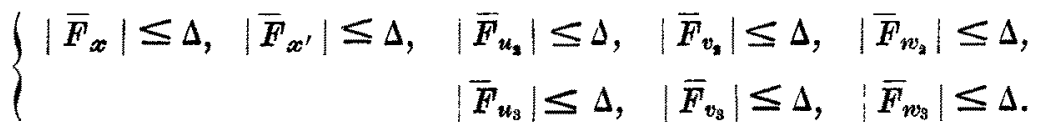

Se invece è

$$
\left|u_{3}\right|+\left|v_{3}\right|+\left|w_{3}\right| \geq H \geq 1,
$$

essendo, in virtù della (22)

$$
1-\frac{\eta}{2}<1+\tau_{r}\left(\frac{1}{D^{\frac{4}{3}}}-1\right)<1+\frac{\eta}{2}
$$

dalle (34), tenendo conto delle (36) e (35), segue

$$
\begin{aligned}
(1-\eta)\left(\left|u_{3}\right|+\left|v_{3}\right|+\left|w_{3}\right|\right)< & \\
<\left|u_{3}+\tau_{Y}\left(u_{8 Y}-u_{3}\right)\right|+\left|v_{3}+\tau_{\Upsilon}\left(v_{3 Y}-v_{3}\right)\right| & +\left|w_{3}+\tau_{Y}\left(w_{3 Y}-w_{3}\right)\right|< \\
& <\left(1+\eta \mid\left(\left|u_{3}\right|+\left|v_{3}\right|+\left|w_{3}\right|\right) .\right.
\end{aligned}
$$

Pertanto, in virtù delle (14) e delle (37), tenendo presente la (13), la (23) e la (31), si può affermare che per $|\gamma|<\gamma_{2}$, ciascuno dei prodotti

$$
\mathbf{Q} \bar{F}_{x}, \Omega^{\prime} \bar{F}_{x^{\prime}}, \frac{u_{2 \gamma}-u_{2}}{\gamma} \bar{F}_{u_{2}}, \frac{v_{2 \gamma}-v_{2}}{\gamma} \bar{F}_{v_{2}}, \frac{w_{2 \gamma}-w_{2}}{\gamma} \bar{F}_{w_{*}}
$$

che figurano al secondo membro della (27) risulta integrabile in $\left(p_{0}, p_{3}\right)$.

Per dimostrare che anche ciascuno degli altri tre prodotti è integrabile in tale intervallo, osserviamo che dalle (33) segue, ricordando l'espressione di $T_{1}, T_{2}, T_{3}$,

$$
\left\{\begin{array}{l}
\frac{u_{3 \gamma}-u_{3}}{\gamma}=\frac{u_{3}}{\gamma D^{\frac{4}{3}}}\left(1-D^{\frac{4}{3}}\right)+\frac{T_{1}}{\gamma} \\
\frac{v_{8 \gamma}-v_{3}}{\gamma}=\frac{v_{3}}{\gamma D^{\frac{4}{3}}}\left(1-D^{\frac{4}{3}}\right)+\frac{T_{2}}{\gamma} \\
\frac{w_{8 \gamma}-w_{3}}{\gamma}=\frac{w_{3}}{{ }^{4} D^{\frac{4}{3}}}\left(1-D^{\frac{4}{3}}\right)+\frac{T_{3}}{\gamma}
\end{array}\right.
$$


ed anche, essendo

$$
1-D^{4}=1-\left[1+2 \gamma \boldsymbol{Q}^{\prime}\left(2 x^{\prime}+\gamma Q^{\prime}\right)+\gamma^{\prime} \mathbf{Q}^{\prime 2}\left(2 x^{\prime}+\gamma^{Q^{\prime}}\right)^{2}\right]
$$

$$
\left\{\begin{array}{l}
\frac{u_{3 \gamma}-u_{3}}{\gamma}=-\frac{u_{3}}{D^{\frac{4}{3}}}\left(4 x^{\prime} \Omega^{\prime}+\chi(\gamma)\right)+\frac{T_{1}}{\gamma} \\
\frac{v_{3 \gamma}-v_{3}}{\gamma}=-\frac{v_{3}}{D^{\frac{4}{3}}}\left(4 x^{\prime} \Omega^{\prime}+\chi(\gamma)\right)+\frac{T_{2}}{\gamma} \\
\frac{w_{3 \gamma}-w_{3}}{\gamma}=-\frac{w_{3}}{D^{\frac{4}{3}}}\left(4 x^{\prime} \Omega^{\prime}+\chi(\gamma)\right)+\frac{T_{3}}{\gamma},
\end{array}\right.
$$

dove $X(\gamma)$ è limitato in $\left(p_{0}, p_{3}\right)$ ed infinitesimo per $\gamma \rightarrow 0$.

Pertanto si puo affermare, tenendo presenti la (36), la seconda delle (20) e la (22), che esistono due numeri positivi $\Lambda_{1}$ e $\Lambda_{2}$ tali che, per $|\gamma|<\gamma_{2}$, è

$$
\left.\left|\frac{u_{3 \gamma}-u_{3}}{\gamma}\right|+\left|\frac{v_{3 \gamma}-v_{3}}{\gamma}\right|+\left|\frac{w_{3 \gamma}-w_{3}}{\gamma}\right| \leq \Lambda_{1}|| u_{3}|+| v_{3}|+| w_{3} \mid\right\}+\Lambda_{2},
$$

e quindi, in virtù dell' altima delle (14), delle ultime tre delle (18) e delle ultime tre delle (37), ciascuno dei prodotti

$$
\frac{u_{3 \gamma}-u_{3}}{\gamma} \bar{F}_{u_{3}}, \quad \frac{v_{3 \gamma}-v_{3}}{\gamma} \bar{F}_{v_{3}}, \quad \frac{w_{3 \gamma}-w_{3}}{\gamma} \bar{F}_{n_{3}}
$$

risulta integrabile in $\left(p_{0}, p_{3}\right)$. Dunque esiste finito $l^{\prime}$ integrale $\mathfrak{g}_{C_{\gamma}^{(3)}}^{(3)}$, ed è, tenendo presenti le (21) e le (26), e ricordando la (27),

$$
\begin{aligned}
& \text { (40) } \frac{\mathfrak{J}_{\gamma}^{(3)} C_{\gamma}^{(3)}-\mathfrak{J}_{C_{0}^{(3)}}^{(3)}}{\gamma}=\int_{p_{0}}^{p_{8}} \mid \Omega \bar{F}_{x}+\Omega^{\prime} \bar{F}_{x^{\prime}}+\frac{u_{2 \gamma}-u_{2}}{\gamma} \bar{F}_{u_{\mathbf{z}}}+\frac{v_{2 \gamma}-v_{2}}{\gamma} \bar{F}_{v_{\mathbf{z}}}+\frac{w_{2 \gamma}-w_{2}}{\gamma} \bar{F}_{n_{\mathbf{z}}}+ \\
& \left.+\frac{u_{3 \gamma}-u_{3}}{\gamma} \bar{F}_{u_{3}}+\frac{v_{3 \varphi}-v_{3}}{\gamma} \bar{F}_{v_{3}}+\frac{w_{3 \gamma}-w_{3}}{\gamma} \bar{F}_{w_{3}}\right] d s .
\end{aligned}
$$

Osserviamo che, poichè è $\lim _{\gamma \rightarrow 0} D=1$ e, tenendo presente la (30),

$$
\lim _{\gamma \rightarrow 0} \frac{1-D^{2}}{\gamma}=-6 x^{\prime} \Omega^{\prime}
$$


dalle (29) segue

$$
\left\{\begin{array}{l}
\lim _{\gamma \rightarrow 0} \frac{u_{2 \gamma}-u_{2}}{\gamma}=-\left(2 x^{\prime} u_{2}+z^{\prime} v_{2}\right) \Omega^{\prime}-y^{\prime} \Omega^{\prime \prime} \\
\lim _{\gamma \rightarrow 0} \frac{v_{2 \gamma}-v_{2}}{\gamma}=-3 x^{\prime} v_{2} \Omega^{\prime} \\
\lim _{\gamma \rightarrow 0} \frac{w_{2 \gamma}-w_{2}}{\gamma}=-\left(2 x^{\prime} w_{2}+y^{\prime} v_{2}\right)+z^{\prime} \Omega^{\prime \prime}
\end{array}\right.
$$

inoltre, tenendo presente che dalla $\left(37^{\prime}\right)$ si ha

$$
\lim _{\gamma \rightarrow 0} \frac{1-D^{\frac{4}{3}}}{\gamma}=-4 x^{\prime} \mathbf{Q}^{\prime}
$$

dalle (33) segue

$$
\left\{\begin{array}{l}
\lim _{\gamma \rightarrow 0} \frac{u_{3 \gamma}-u_{3}}{\gamma}=-\left[3 x^{\prime} u_{3}+z^{\prime} v_{3}+\frac{1}{R^{2}} y^{\prime}+3\left(z^{\prime} w_{2}-y^{\prime} u_{2}\right) u_{2}\right] \Omega^{\prime}-3 x^{\prime} u_{2} \Omega^{\prime \prime}-y^{\prime} \Omega^{\prime \prime \prime} \\
\lim _{\gamma \rightarrow 0} \frac{v_{8 \gamma}-v_{3}}{\gamma}=-\left[4 x^{\prime} v_{3}+3\left(z^{\prime} w_{2}-y^{\prime} u_{2}\right) v_{2}\right] \Omega^{\prime}-3 x^{\prime} v_{2} \Omega^{\prime \prime} \\
\lim _{\gamma \rightarrow 0} \frac{w_{3 \gamma}-w_{3}}{\gamma}=-\left[3 x^{\prime} w_{3}+y^{\prime} v_{3}-\frac{1}{R^{\prime}} z^{\prime}+3\left(z^{\prime} w_{2}-y^{\prime} u_{2}\right) w_{2}\right] \Omega^{\prime}-3 x^{\prime} w_{2} \Omega^{\prime \prime}+z^{\prime} \mathbf{Q}^{\prime \prime \prime}
\end{array}\right.
$$

Allora la funzione integranda, che figura al secondo membro della (40), per $\gamma \rightarrow 0$ tende in quasi tatto $\left(p_{0}, p_{3}\right)$ a

$$
\begin{gathered}
Q F_{x}+\left\{F_{x^{\prime}}-\left(2 x^{\prime} u_{2}+z^{\prime} v_{2}\right) F_{u_{2}}-3 x^{\prime} v_{2} F_{v_{3}}-\left(2 x^{\prime} w_{2}+y^{\prime} v_{2}\right) F_{n_{2}}-\right. \\
-\left[3 x^{\prime} u_{3}+z^{\prime} v_{3}+\frac{1}{R^{2}} y^{\prime}+3\left(z^{\prime} w_{2}-y^{\prime} u_{2}\right) u_{2}\right] F_{u_{3}}-\left[4 x^{\prime} v_{3}+3\left(z^{\prime} w_{2}-y^{\prime} u_{2}\right) v_{2}\right] F_{v_{3}}- \\
-\left[3 x^{\prime} w_{3}+y^{\prime} v_{3}-\frac{1}{R^{2}} z^{\prime}+3\left(z^{\prime} w_{2}-y^{\prime} u_{2}\right) w_{2}\right] F_{w_{3}} \Omega^{\prime} \Omega^{\prime}+ \\
+\left[z^{\prime} F_{w_{z}}-y^{\prime} F_{u_{3}}-3 x^{\prime}\left(u_{2} F_{u_{3}}+v_{2} F_{v_{3}}+w_{2} F_{w_{3}}\right)\right] \Omega^{\prime \prime}+\left(z^{\prime} F_{w_{3}}-y^{\prime} F_{u_{8}} \Omega^{\prime \prime \prime} .\right.
\end{gathered}
$$

Inoltre, tenuto presente che, in virtù della (31), e ricordando la (24), esiste un numero positivo $G_{0}$ tale che, per $|\gamma|<\gamma_{2}$, è

$$
\left|\frac{u_{2 \gamma}-u_{2}}{\gamma}\right|+\left|\frac{v_{2 \zeta}-v_{2}}{\gamma}\right|+\left|\frac{w_{2 \gamma}-w_{2}}{\gamma}\right|<G_{0},
$$


e tenuto conto delle (20) e (39), delle (14), (18) e (37), la funzione integranda, che dal secondo menubro della (40), risulta in valore assoluto minore di

$$
\left|\frac{\left.p_{3}-p_{0}\right)^{3}}{3 !}+\frac{\left(p_{3}-p_{0}\right)^{2}}{2}+3\left(G_{0}+\Lambda_{1} H+\Lambda_{2}\right)\right|\left(\Xi_{3}+\Delta\right) \text {. }
$$

Si conclude pertanto chə è, tenendo presenti le (4),

$$
\begin{aligned}
\lim _{\gamma \rightarrow 0} & \frac{\mathfrak{J}_{C_{\gamma}^{(s)}}^{(s)}-\mathfrak{J}_{C^{(3)}}^{(3)}}{\gamma}= \\
& =\int_{p_{0}}^{p_{3}} \int_{1} \Omega F_{x}+\left[F_{x^{3}}-v_{2} \Phi_{2}-2 x^{\prime} \Psi_{22}-v_{3} \Phi_{3}-3 x^{\prime} \Psi_{s 3}-3\left(z^{\prime} w_{2}-y^{\prime} u_{2}\right) \Psi_{23}-\right. \\
& \left.\left.-\frac{1}{R^{2}}\left(y^{\prime} F_{u_{3}}-z^{\prime} F_{w_{3}}\right)\right] \Omega^{\prime}+\left[z^{\prime} F_{w_{s}}-y^{\prime} F_{u_{2}}-3 x^{\prime} \Psi_{23}\right] \Omega^{\prime \prime}+\left(z^{\prime} F_{v_{3}}-y^{\prime} F_{u_{3}}\right) \Omega^{\prime \prime \prime}\right\} d s
\end{aligned}
$$

ed il resto della dimostrazione procede in modo noto $\left({ }^{13}\right)$.

In modo analogo si giunge a stabilire la seconda e la terza delle (5). del n. 3 .

Osservazione. - Si possono fare due osservazioni analoghe a quelle

7. Casi particolari. - Si possono rilevare, per il teorema del n. 6, casi particolari analoghi a quelli considerati al n. 4. Per quanto riguarda il caso analogo a quello che forma oggetto del capoverso b) del n. 4 , è ovvio che nell'enunciato deve figurare anche il numero $M_{0}$ che interviene nel teorema del n. 6, in modo che le disuguaglianze analoghe alle (11) e (12) siano, in questo caso, verificate in tutti i punti $(x, y, z)$ di $A_{\lambda}$, per ogni terna $x^{\prime}, y^{\prime}, z^{\prime}$ normalizzata, per ogni terna di numeri reai i $u_{2}, v_{2}, w_{2}$ per cui $u_{2}^{2}+v_{2}^{2}+w_{2}^{2} \leq M_{0}^{z}$, e per qualunque terna di numeri reali $u_{3}, v_{s}, w_{s}$.

8. Esempi. - Le funzioni

$$
\begin{aligned}
& F=\sqrt{x^{\prime 2}+y^{\prime 2}+z^{\prime 2}}\left(u_{3}^{2}+v_{3}^{2}+w_{3}^{2}\right) e^{\frac{1}{1+u_{3}^{2}+v_{3}+w_{3}^{2}}}, \\
& F=\sqrt{x^{\prime 2}+y^{\prime 2}+z^{\prime 2}}\left(u_{3}^{2}+v_{3}^{2}+w_{3}^{2}\right)^{2} \log \left(1+u_{3}^{2}+v_{3}^{2}+w_{3}^{2}\right), \\
& F=\sqrt{x^{\prime 2}+y^{\prime 2}+z^{\prime 2}} \sqrt[n]{1+\left(u_{3}^{2}+v_{3}^{2}+w_{3}^{2}\right)^{\prime \prime \prime}}, \\
& F=\sqrt{x^{2}+y^{\prime 2}+z^{\prime 2}} \sqrt[n]{1+u_{3}^{2 m}+v_{3}^{2 m}+w_{3}^{2 m}},
\end{aligned}
$$

dove $m$ ed $n$ sono interi positivi, soddisfano alle condizioni del n. 6, come si pud verificare in modo analogo al n. 5 .

${ }^{\left(3^{\prime}\right)}$ Vedi S. Cinquini, Sopra le equazioni di Eulero dei problemi variazionali di ordine $n$, a Annali di Matematica pura ed appplicata ^, S. IV, T. XVI (1937), pp. 61-100. Cfr. § 2, n. 7. 
9. Due condizioni sufficienti affinchè un' estremaloide di ordine 2 sia un' estremale di ordine 2.

10) Se la (2) è un' estremaloide di ordine 2, per la quale esistono finite e continue in tutto $(0, L)$ le derivate $x^{\prime \prime}(s), y^{\prime \prime}(s), z^{\prime \prime}(s)$, allora la (2) è anche estremale di ordine 2.

Ciò è immediato; dalle (3) segue infatti, per ogni $s$ di $(0, L)$,

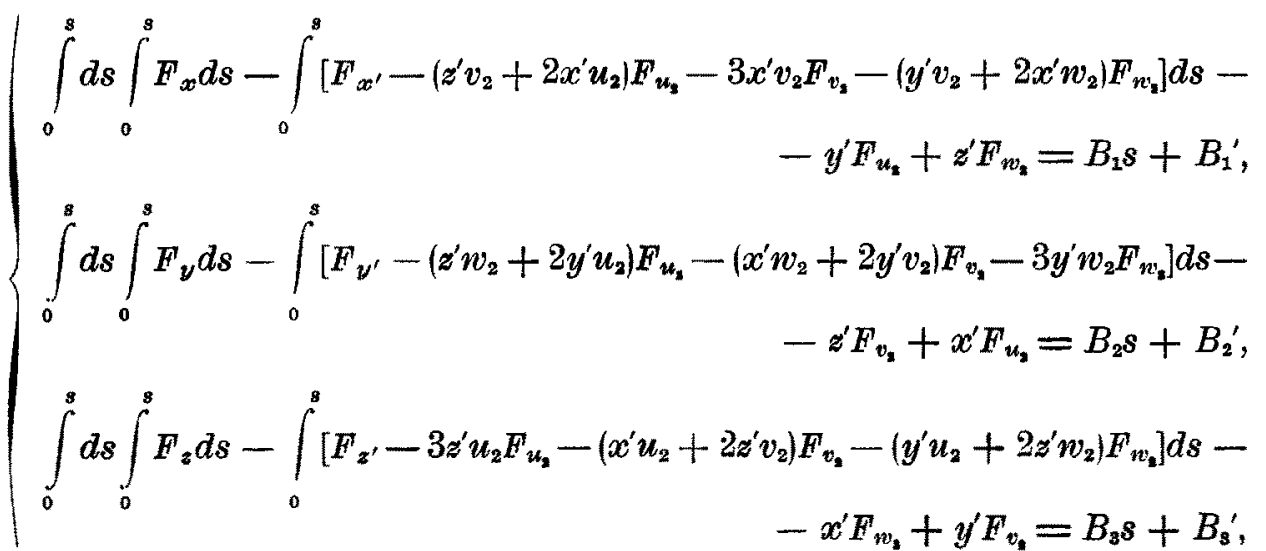

per cui, tenuto conto del risultato conseguito al n. 2 della Memoria citata in ('), l'asserto è dimostrato.

$\left.2^{\circ}\right)$ Se $\mathfrak{J}_{C^{(2)}}^{(2)}$ è un integrale quasi-regolare positivo normale, ogni estremaloide di ordine 2 , per la quale le derivate $x^{\prime}(s), y^{\prime}(s), z^{\prime}(s)$ sono a rapporto incrementale limitato, è un'estremale di ordine 2.

Per dimostrare l'asserto estendiamo con opportuni accorgimenti un pro. cedimento seguito da ToNeLLI $\left({ }^{\mathbf{1 4}}\right)$.

Indichiamo con $E$ il pseudointervallo costituito dai valori di $(0, L)$ nei quali esistono finile le derivate $x^{\prime \prime}(s), y^{\prime \prime}(s), z^{\prime \prime}(s)$, e ciascuno degli integrali

$$
\int_{0}^{s}\left(y^{\prime} F_{u_{2}}-z^{\prime} \bar{F}_{w_{2}}\right) d s, \quad \int_{0}^{8}\left(z^{\prime} F_{v_{2}}-x^{\prime} F_{u_{2}}\right) d s, \quad \int_{0}^{8}\left(x^{\prime} F_{w_{2}}-y^{\prime} F_{v_{2}}\right) d s
$$

ammette come derivata la funzione integranda, tenendo presente che per ogni valore di $E$ sono verificate le (41).

Sia ora $s$ un valore di $(0, L)$ non appartenente ad $E$; osservato che,

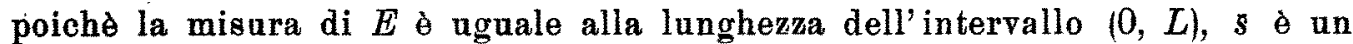
valore di accumulazione di punti di $E$, dimostriamo che al tendere di un

(14) Vedi luogo cit. in ( $\left.{ }^{4}\right)$, n. $34 d$ ), pp. 101-108. 
valore qualunque $s$ di $E$ ad $\bar{s}$ ognuna delle funzioni $u_{2}, v_{2}, w_{2}$ tende ad un unico valore.

Si supponga infatti che al tendere di $s$ ad $\bar{s}$ si abbiano per (una almeno tra) $u_{2}, v_{2}, w_{2}$ almeno due valori di accumulazione distinti, e siano questi rispettivamente $\bar{u}_{2}, \overline{\bar{u}}_{2}, \bar{v}_{2}, \overline{\bar{v}}_{2}, \bar{w}_{2}, \overline{\bar{w}}_{2}$, nel senso cioè che non valgano simultaneamente le tre uguaglianze $\bar{u}_{2}=\overline{\bar{u}}_{2}, \bar{v}_{2}=\overline{\bar{v}}_{2}, \bar{w}_{2}=\overline{\bar{w}}_{2}$.

Indichiamo, per brevità, con $\bar{x}, \bar{y}, \bar{z}, \bar{x}^{\prime}, \bar{y}^{\prime}, \bar{z}^{\prime}$ rispettivamente le funzioni $x(s), y(s), z(s)$ e le loro derivate del primo ordine calcolate in $s$, e con $\bar{F}_{w_{2}}, \bar{F}_{v_{2}}, \bar{F}_{w_{2}}, F_{w_{2}}, \overline{\bar{F}}_{v_{2}}, \overline{\bar{F}}_{w_{3}}$ le derivate parziali $F_{w_{2}}, F_{v_{2}}, F_{w_{2}}$ ealcolate rispettivamente in $\left.\overline{(x}, \bar{y}, \bar{z} ; \bar{x}^{\prime}, \overline{y^{\prime}}, \bar{z} ; \bar{u}_{2}, \bar{v}_{2}, \bar{w}_{2}\right)$ e in $\left(x, \bar{y}, \bar{z} ; \bar{x}^{\prime}, \bar{y}^{\prime}, \bar{z}^{\prime} ; \overline{\bar{u}}_{2}, \overline{\bar{v}}_{2}, \bar{w}_{2}\right)$.

Dalle $(41)$, per $s=\bar{s}$, segue

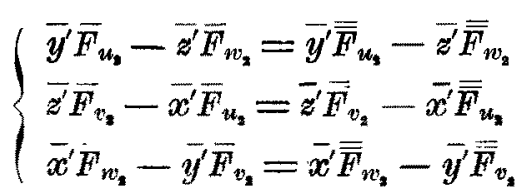

e anche, moltiplicando ambo i membri della prima, seconda e terza delle (42) rispettivamente per

$$
\bar{x}^{\prime \prime}=\bar{z}^{\prime} \bar{w}_{2}-\bar{y}^{\prime} \bar{u}_{2}, \quad \bar{y}^{\prime \prime}=\bar{x}^{\prime} \bar{u}_{2}-\bar{z}^{\prime} \bar{v}_{2}, \quad \overline{z^{\prime \prime}}=\overline{y^{\prime}} \bar{v}_{2}-\bar{x}^{\prime} \bar{w}_{2}
$$

e sommando membro a membro, e moltiplicando successivamente per

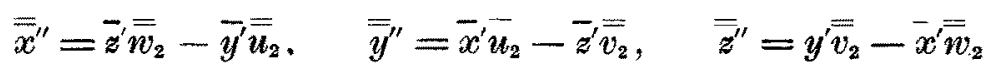

e ancora sommando membro a membro,

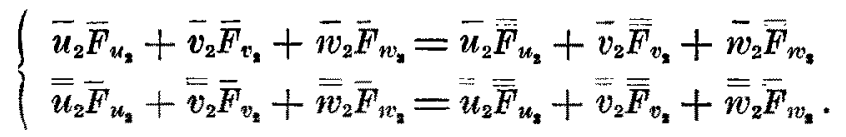

Dalle (43) si ha dunque

$$
\left(\bar{u}_{2}-\overline{\bar{u}}_{2}\right)\left(\bar{F}_{u_{2}}-\overline{\bar{F}}_{u_{2}}\right)+\left(\bar{v}_{2}-\overline{\bar{v}}_{2}\right)\left(\bar{F}_{v_{2}}-\overline{\bar{F}}_{v_{2}}\right)+\left(\bar{w}_{2}-\overline{\bar{w}}_{2}\right)\left(\bar{F}_{w_{2}}-\overline{\bar{F}}_{w_{2}}\right)=0 ;
$$

d'altra parte, poichè abbiamo supposto l'integrale $\mathfrak{J}_{C^{(2)}}^{(2)}$ quasi-regolare positivo normale, è, posto per brevità

$$
\begin{gathered}
\bar{F}=F\left(\bar{x}, \bar{y}, \bar{z} ; \bar{x}^{\prime}, \bar{y}^{\prime}, \bar{z}^{\prime} ; \bar{u}_{2}, \bar{v}_{2}, \bar{w}_{2}\right) \\
\overline{\bar{F}}=F\left(\bar{x}, \bar{y}, \bar{z} ; \bar{x}^{\prime}, \bar{y}^{\prime}, \bar{z}^{\prime} ; \overline{\bar{u}}_{2}, \overline{\bar{v}}_{2}, \overline{\bar{w}}_{2}\right), \\
\overline{F^{\prime}}-\overline{\bar{F}}-\left(\bar{u}_{2}-\overline{\bar{u}}_{2}\right) \overline{\bar{F}}_{u_{z}}-\left(\overline{\bar{v}}_{2}-\overline{\bar{v}}_{2}\right) \overline{\bar{F}}_{v^{\prime}}-\left(\bar{w}_{2}-\overline{\bar{w}}_{2}\right) \overline{\bar{F}}_{w_{2}}>0 \\
\overline{\bar{F}}-\bar{F}-\left(\overline{\bar{u}}_{2}-\bar{u}_{2}\right) \bar{F}_{u_{z}}-\left(\overline{\bar{v}}_{2}-\bar{v}_{2}\right) \bar{F}_{v_{2}}-\left(\overline{\bar{w}}_{2}-\bar{w}_{2}\right) \bar{F}_{w_{1}}>0,
\end{gathered}
$$


e quindi anche

$$
\left.\left(\bar{u}_{2}-\overline{\bar{u}}_{2}\right)\left(\bar{F}_{u_{t}}-\overline{\bar{F}}_{u_{2}}\right)+\left(\bar{v}_{2}-\overline{\bar{v}}_{2}\right)\left(\bar{F}_{v_{2}}-\overline{\bar{F}}_{v_{2}}\right)+\left(\bar{w}_{2}-\overline{\bar{w}}_{2}\right) \mid \bar{F}_{i_{2}}-\overline{\bar{F}}_{v_{2}}\right)>0
$$

in contrasto con la (44).

Pertanto al tendere di $s$ ad $s$ ognuna delle funzioni $u_{2}, v_{2}, w_{2}$ tende ad un unico valore, e l'asserto è dimostrato.

10. Due condizioni sufficienti affinchè un'estremaloide di ordine 3 sia un' estremale di ordine 3 .

10) Se la (2') è un' estremaloide di ordine 3, per la quale esistono finite $e$ continue in tutto $(0, L)$ le derivate $x^{\prime \prime \prime}(s), y^{\prime \prime \prime}(s), z^{\prime \prime \prime}(s)$, allora la $\left(2^{\prime}\right)$ è anche un estremale di ordine 3 .

L'asserto si prova immediatamente ragionando come al n. 9, $1^{\circ}$ ).

$2^{\circ}$ Se $\mathfrak{J}_{C^{(3)}}^{(3)} \dot{e}$ un integrale quasi-regolare positivo normale, ogni estremaloide di ordine 3 per la quale le derivate $x^{\prime \prime}(s), y^{\prime \prime}(s), z^{\prime \prime}(s)$ sono a rapporto incrementale limitato, è un' estremale di ordine 3 .

Per dimostrare quanto si è affermato si procede in modo del tutto analogo a quello seguito nel capoverso $2^{\circ}$ ) del n. 9 .

Ci limitiamo a rilevare che, in luogo della somma $u_{2} F_{u_{z}}+v_{2} F_{v_{3}}+w_{3} F_{w_{z}}$ che ha ruolo importante nella dimostrazione relativa al caso del secondo ordine, figura, in questo caso, la somma $u_{3} F_{u_{3}}+v_{3} F_{v_{3}}+w_{3} F_{v_{3}}$. 